UC-32

Reporting Date: April 1975

Issued: July 1975

\title{
A Method of Characteristics for the Transport Equation Solution
}

by

Bengt G. Carlson

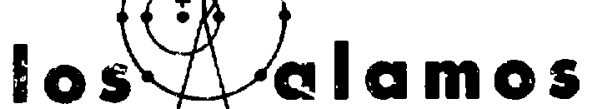

scientific laboratory

of the University nf California

los alamos, NeW MEXico 87545

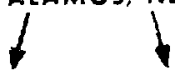

An Affirmative Action / Equal Opportunity Employer 
Printed in the United States of America. Available from Nationd Technical Information Service U S Department of Commerce

$$
\begin{aligned}
& 5285 \text { Port Royal Roud } \\
& \text { Springfield, VA } 22151
\end{aligned}
$$

Price: Printed Copy $\$ 4.00$ Microfiche \$2.25

This report was prepared as an eccount of work sponsored

b3 the Cnited States Governmeat. Neither the United States

nor the Uniled States Enerey Research and Development Ad.

ministration, nor anv of their employees, nor any of their con.

tractors, suberniractors or their employeen, makes any

responsibitity for the gecuracy. completenens. or unefulness uf

anv information, apparitus, product, or process dieclosed, of

represents that its ure would nat infrinte privately owned 


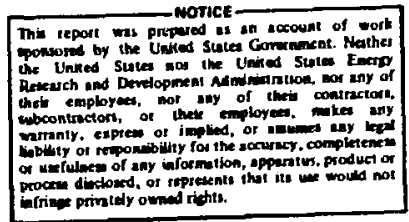

A METHOD OF CHARACTERISTICS FOR THE TRANSPORT EQUATION SOLUTION

by

Bengt G. Car1son

\section{ABSTRAC'I}

A method of characteristics is developed for the numerical solution of the multigroup discrete ordinates transport equacion. A revised form of the diamond difference method is coordinated with the new approach and some alternate, possibly useful methods are developed. Also, several minor numerical difficulties that occasionally troubled calculations in the pasc have been resolved.

\section{INTRODUCTION}

Th1s report discusses the numerical solution of the multigroup, discrete ordinates transport equation with special emphasis on neutron transport and diffuston, and develops an improved $s_{n}$-type method for such solution with several new features. Included among these is a method of characteristics that furnishes a differant approach to the solution. For clarffication, a transport method is a collection of methods, which typically includes discretizations, quadrature formulas, difference schemes, and iterative techniques, plus numerous special methods for special situations. Reference 1 should be regarded as a companion report to this one. To some extent, this report updates and rearrauges material from Ref. 1.

The revised methods have many advantages over earlier methods, which will become apparent as the discussion progresses. The characteristic approach, for example, Impaxts more general and consistent properties to the final discretized equations. It facilitates the generation of physically realistic solutions even under very adverse conditions. Specffically, the positivity and smoothness of a solutIon can be inforced as the computations progress, which reduces or eliminates various types of oscillations, Including, to some degree, so-called ray effects. This, in turn, has a beneficlal effect on the convergence rate of iterative sequences. However, the revised method is not radically different from the $S_{n}$ methods and codes now used, or from those explored or used in the past.

Solution by a discrete $S_{n}$ (DSN) method typically involves plecing together a distribution function over a coordinate mesh in energy, direction, time, and posftion by means of straight-line segments, using continuous segments whenever this seems che most sensible thing to do. Based on various pract1cal considerations, the discrete treatment has become the favored treatment for the energy varfable. In other variables, including directional or angular, the normal treatment is by continuous segments, with occasional departures from that norm by means of scicalled modified difference schemes. The finite ele-ment and spherical harmonic methods represent significant departures from the general approach of this report. The finite element method replaces simple difference schemes by more elaborate techniques, and the spherical harmonics method approximates the angular-dependent segment of the distribution by a continuous polynomial rather than by a net of connected straight-line segments.

The multigroup, discrete ordinates equation is a simplified form of the linear Boltzmann equation, an integro-differential equation. It governs the distribution $\mathrm{N}$ of particles, typically neutror

1

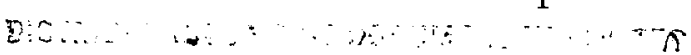


photons, as a function of particle class $(g, m)$ location In space $R$, and time $t$, and consists of a system of $G$ times $M$ partial differential equations of the form

$$
\frac{\partial}{\partial s} N_{g, m}(s)+\sigma_{g}(s) N_{g, m}(s)=s_{g}(s),
$$

where $g=1,2, \ldots, G$, and $m=1,2, \ldots, M$. The functions $\mathrm{N}_{\mathrm{g}, \mathrm{m}}$ are the unknowns to be determined, given the other quantities. Here an Index pair $(g, m)$ refers to a beam or collection of neutrons, say, of velocity about $v_{g}$ moving in the direction about $\Omega_{m}$. The quantity $s$ is measured along so-called characteristic lines, that is, in the direction $\Omega_{\mathrm{m}}$ of streamIng. Equation (1.1) assumes that time $t$ and the components of $R$ depend parametrically on $s$.

The multigroup transformation is largely a transformation of the physical data (mainly crosssection data for a variety of particle-nuclei interactions) from their more natural energy-dependent form to the multigroup or transfer matrix form. When done to any precision, this is a very complex and laborlous transformation. The data include the total removal cross-section $\sigma_{g}$ shown explicitly in Eq. (1.1) and simflar, more detailed data used to form the source term $s_{g}$. This term is the principal coupling term in the above system of $G$ times $M$ equations.

For any given $g, S_{g}$ generally depends on the $\mathrm{N}_{\mathrm{g}, \mathrm{m}}$ for that $\mathrm{g}$, as well as on $\mathrm{N}_{\mathrm{g}, \mathrm{m}}$ for other or perhaps all g. Therefore, to arrive at a numerical solution in a simple way, a sequence of iterative cycles is performed. Inicinlly, $s_{g}$ is computed from given trial functions but at later stages it comes from previous 1terates. Fortunately, in most practical situations, such sequences of iterates converge. Moreover, in cases of slow convergence, various means are avallable for the acceleration of convergence.

The solution for $\mathrm{N}$ in angle, space, and time is obtafned by means of difference schemes. For angular components, quadrature methods are also involved. The solution is generated mesh cell by mesh cell in the $(\Omega, R, t)$ space, usually in a very particular sequence. Iteration is invollved for each time step and each group $g$. Here, $R$ may have up to three components, and $\Omega \equiv(\mu, \eta, \xi)$ with $\mu^{2}+\eta^{2}+\xi^{2}=1$, up to two Independent components. Assuming regular mesh cells with d dimensions and two times d sides, the calculations irst encafl the determination of the midcell value of $\mathrm{N}$ and then $\mathrm{d}$ extrapolation across the cell. The latter, which may be based on the $d$ input fluxes and the midcell flux, determine the cell output fluxes. Here by extrapolation scheme is meant the same as difference scheme. In the rectangular geomeirles (the Cartesian case), with $R=(x, y, z)$ In the most general case, extrapolation In the angular varfables is normally not necessary. In the simple curvtlinear cases of spherical ( $r$ ) and cylindrical geometry, only one extrapolation in angle is needed. Discontinuous extrapolation, used occaslonally, is used chlefly to maintain positive output fluxes.

In the discrete $S_{n}$ method, one normally chooses $M_{0}=n(n+2) / 8$ directions per octant of the unit sphere, the general domain of $\Omega$, where $n$ is the order of angular approximation, $n=2,4, \ldots$. The usual value of $M$ is therefore $2 M_{0}$, where $d$ is the dimensionality of $R$. The calculations are greatly simplifled if the total mesh in all the variables present is obtained as the logical product of the individual meshes. Although this restriction is conventent and generally reduces the calculation time per cell, it is not necessary, may not suit all applications, and may produce far too many cells.

The revised methods can be expected to save a great deal of computing time because, with smoother fluxes'expected, the number of cells in one or several of the varlables may be decreased markedly and the number of iterations needed for convergence may also be reduced. The basic procedure ises special schemes in a variety of situations. In general, such schemes are more claborate than the regular ones. However, the speclial schemes are only used when actually needed and involve but a fraction of the cells, this having little effect on the calculation time. The fraction depends on the locality in the configuration and on the cell sizes chosen, as well as on the particle speed and direction.

The main difficulty with the discrete ordinate, difference relaition approach is the occasional development of flux distortions, often in the form of oscillations in the calculated fluxes with no basis in the physics of the problem. Osctilations in the forward direction (flux seesaw), osclllations at large angles to the main stream (flux skewing), and Irregular, wave-like varfations in the flux referred to as ray effects can be observed. 
Oscillations generally develop as a consequence of rapid local varlation in the $f$ ? $1 \mathrm{x}$ with too large Intervals and tou stiff extrapolations to handle the situation. In this context, the importance of the optical dimensions that depend on cross-sectious $\sigma_{g}$ multiplfed by cell dimensions is noted. Actually, as explained later, what counts is the effective optical jimensions. Oscillations can sometimes be controlled by inserting more intervals in the approprlate places. In such localities, however, they are better controlled by usitig modified difference schemes if a precise determination of the flux is not of pritpe concern.

Ray effects develop in certain situations as a result of the discrete ordinates approximation in the absence of sufictent natural smoothing, for example, by particle scattering. Ray effects can be mitigated readily by increasing the order $n$ but, unfortunately, the iitigation tends to go slowly with increasing $n$. They can be effectively reduced or ellminated by introducing a Eictitious source correction of total strength zero, which operates as a smoothing agent. In general, well-based, effective source corrections are very complex and are expensive in terms of computer time and storage. Also, they interfere seriously with the rate of iterative convergence. Other smoothing methods combined with a simplified fictitious source, as suggested in this report, may possibly be an answer to this occastonal dilemna.

\section{RECTANGULAR GEOMETRIES}

For $(x, y)$ rectangular geometry, the multigroup transport equation is given by section, denotes the probability per unit length of travel that a given particle experiences a collision (a change in $\Omega$, a removal from the beam). The source term $\mathrm{S}_{\mathrm{g}}$ denotes the number of particles of velocity about $v_{B}$ being released (inserted into the beam) per unit volume at $(x, y)$ per unit time. Therefore, $V \sigma_{g} N_{g}$ represents the Interaction losses in a small volume $V$ at $(x, y)$, VS $g$ the interaction and other gains, and $\mathrm{V}\left(\mathrm{S}_{\mathrm{g}}-\sigma_{\mathrm{g}} \mathrm{N}_{\mathrm{g}}\right)$ the net change due to all local sources and sinks. In particular, the term $\mathrm{s}_{\mathrm{g}}$ acts as a coupling term between the groups. It includes contributions $f_{\text {rom }}$ its own and other groups, frow a selection of the groups if not from all. In the neutron case, it includes contributions from independent sources, scattering events, and fission reactions. The derfvative terms in Eq. (2.1), howevar, have to do with the migration or propagation of particles in the $(x, y)$ space.

Equation (2.1) illustrates the general rectangular case that may include as many as four derivative terms. Equation (2.1) is simplified to one less term if $\mathrm{N}_{\mathrm{g}}$ depends on $\mathrm{x}$ only. It gains a term $\xi\left(\partial N_{g} / \partial z\right)$ if $N_{g}$ depends on $z$ as well as on $x$ and $y$. Finally, it recelves a term $\partial \mathrm{N}_{g} / \mathrm{v}_{g}$ at if $\mathrm{N}_{g}$ is also a function of time $t$.

Equation (2.1) can be written more concisely In the characteristic form

$$
\frac{\partial}{\partial s} \mathrm{~N}(s, \Omega)+\sigma(s) N(s, \Omega)=\mathrm{s}(\mathrm{s}, \Omega)
$$

where the variable $s$ is measured along the path determined by $\Omega$, with $\Omega$ assuming the role of a

$$
\mu \frac{\partial}{\partial x} N_{g}(x, y, \mu, \eta)+\eta \frac{\partial}{\partial y} N_{g}(x, y, \mu, \eta)+\sigma_{g}(x, y) N_{g}(x, y, \mu, \eta)=s_{g}(x, y) .
$$

This is the analytical equation discretized in the energy varlable. If the particles are neutrons, discrete energy is represented by a set of $G$ discrete velocities $v_{g}, g=1,2, \ldots, G$. The simple fortn of Eq. (2.1) also reflects that isotropic scattering, isotropic sources, and a stationary situation have been asstimed.

In the above, $\mathrm{N}_{\mathrm{g}}$ mears the number of particles of velocity about $v_{g}$ streaming in the direction $\Omega$, $\Omega \equiv(\mu, \eta, \xi)$ with $\mu^{2}+\eta^{2}+\xi^{2}=1$, per unit area normal to $\Omega$ at $R, R \equiv(x, y)$, per unit time. The quantity $\sigma_{g}$, called the total macroscopic cross parameter. Here and following, the index $g$ is suppressed. Equation (2.2) is the physically more meaningful equation that describes the attenuation and amplification of the intensity or flux of a untdirectional beam involving a single independent variable $\mathrm{s}$. Given a change in $\mathrm{s}$, the changes in the other variables can be calculated. Equation (2.1) can be derived from Eq. (2.2) by the operator relation

$$
\frac{\partial}{\partial s}=\mu \frac{\partial}{\partial x}+\eta \frac{\partial}{\partial y},
$$


where $\mu=d x / d s$ and $\eta=d y / d s$.

An important subcase in transport theory is diffusion theory, the result of taking the transport equation to the diffusion theory limt. This may be defined as the limic in which $N$ can be approximated by a linear expression in the angular components

$$
N(x, y, i, n)=\vec{N}(x, y)+3 \mu I(x, y)+3 \eta J(x, y),
$$

where $\bar{N}$ is the scalar flux

$$
N(x, y)=\frac{1}{2 \pi} \int_{0}^{\pi} \int_{-1}^{1} d \eta d \theta N(x, y, \mu, n),
$$

and $I$ and $J$ are the currents in the $x$ and $y$ directions, respectively.

$$
I(x, y)=\frac{1}{2 \pi} \int_{0}^{\pi} \int_{1}^{1} \mu d n d \theta N(x, y, \mu, n),
$$

and

$$
J(x, y)=\frac{1}{2 \pi} \int_{0}^{\pi} \int_{-1}^{1} \eta d \eta d \theta N(x, y, \mu, \eta) .
$$

Here, in the special case of $N \equiv 1, \vec{N}=1$ and $I=$ $J=0$. The azimuthal variable $\theta$ is related to $\mu$ and n by

$$
\mu=\sqrt{1-n^{2}} \cos \theta
$$

The mixed mowent $Q$, a second-order moment needed later, is defined by

$$
Q(x, y)=\frac{1}{2 \pi} \int_{0}^{\pi} \int_{-1}^{1} \mu n d n d \theta N(x, y, \mu, n) .
$$

If Eq. (2.1) is integrated over the $\Omega$ domain three separate times after multiplying the terms by 1 , $3 \mu$, and $3 \eta$, respectively, all times $1 / 2 \pi$, one obtains the balance equation

$$
\frac{\partial}{\partial x} I(x, y)+\frac{\partial}{\partial y} J(x, y)+\sigma(x, y) \vec{N}(x, y)=S(x, y),
$$

the net flux equation in the $\mathrm{x}$ direction

$$
\frac{\partial}{\partial x} \bar{N}(x, y)+3 \sigma(x, y) I(x, y)=0,
$$

and the net flux equation in the $y$ direction

$$
\frac{\partial}{\partial y} \bar{N}(x, y)+30(x, y) J(x, y)=0 .
$$

The last two assume that Eq. (2.4) is substituted in Eq. (2.1) before integration. The above three equations, which may be combined into a single secondorder equation, define diffusion theory.

However, if Eq. $(2.4)$ is not substituted in

Eq. (2.1) before integration, the net flux equations are given by

$$
\frac{\partial}{\partial x} \bar{N}(x, y)+3 \frac{\partial}{\partial y} Q(x, y)+3 \sigma(x, y) I(x, y)=0
$$

and

$$
3 \frac{\partial}{\partial x} Q(x, y)+\frac{\partial}{\partial y} \bar{N}(x, y)+3 \sigma(x, y) J(x, y)=0 .
$$

These two equations, plus Eq. (2.10), are consistent with the expansion

$$
\begin{aligned}
N(x, y, \cdot n)= & \bar{N}(x, y)+3 \mu I(x, y)+3 \eta J(x, y) \\
& +15 \mu \eta Q(x, y) .
\end{aligned}
$$

The above procedure, coupled with the assumption that $Q(x, y) \equiv 0$, is an alternate, second route to diffusion theory.

Note that Eq. (2.15) is not a complete polynomial of second order in $\mu$ and $\eta$ because the terms in $\mu^{2}$ and $n^{2}$ are absent. Stated otherwise, Eq. (2.15) is a polynomial of first order with an excess term in $Q$. Therefore, if one desires not to propagate the excess term, done by writing Eq. (2.1) as

$$
\mu \frac{\partial}{\partial x}(N-15 \mu n Q)+\eta \frac{\partial}{\partial y}(N-15 \mu n Q)+\sigma N=s,
$$

Eqs. (2.10), (2.11), and (2.12) follow without assumIng either Eq. (2.4) or (2.15). In this third approach, diffusion theory is obtained without assuming that $Q(x, v) \equiv 0$.

The next two steps represent successive discretizations of the angular variable $\Omega=(\mu, n, \xi)$ and the postition variable $R=(x, y, z)$. The flrst step is the discrete ordinates transformation and the second step is the finte cell formulation. FollowIng thege ateps with a third major step, difference schemes are introduced to effect the numerical solution. 
The mult, coup discrete ordinates equations are on the same P.. ifical basis as Eq. (2.1) and are fiven by

$$
\begin{aligned}
\mu_{m} \frac{\partial}{\partial x} N_{m}(x, y) & +\eta_{m} \frac{j}{\partial y} N_{m}(x, y)+o(x, y) N_{m}(x, y) \\
& =s\{x, y)
\end{aligned}
$$

where $m$ refers to orie of $M$ discrete directions, $\sigma_{\mathrm{m}}=$ $\left(u_{m}, n_{m}, \xi_{n}\right)$, with

$$
m_{m}^{2}+n_{m}^{2}+\xi_{m}^{2}=1
$$

assumed, $m=1,2, \ldots, M$. The direction cosine $\mu$ is normally attached to the $x$ direction, $n$ to the $y$ direction, and $\xi$ to the $z$ direction. In $(x, y)$ geometry, in which four ociants of the unit sphere, or half of the ? space, is normally involved, $M$ is taken to be

$$
M=n(n+2) / 2 .
$$

This implies $n(n+2) / 8$ directions per octant, where $n$ is the order of angular approximation, $n=2,4$, $\ldots$ and also thac

$$
-1 \leq H_{m}+r_{m} \leq 1,
$$

with $0 \leq \xi_{r_{i}} \leq 1$.

For iscrete ordinates in the diffusion theory limit, raf: writes

$$
\lambda_{m_{i}}(x, y)=\bar{N}(x, y)+3 \mu_{m} I(x, y)+3 \eta_{m} J(n, y){ }_{2}
$$

with

$$
\text { and } \begin{aligned}
\bar{N}(x, y) & =\Sigma_{m} w_{m} N_{m}(x, y), \\
I(x, y) & =\Sigma_{m} w_{m} \mu_{m} N_{m}(x, y), \\
J(x, y) & =\Sigma_{m} w_{m} n_{m} N_{m}(x, y) .
\end{aligned}
$$

For consistency with the analytical case, the followIng conditions must hold for the quadrature sets $\left(w_{m}, \mu_{m}, \eta_{m}\right)$.

$$
\Sigma_{m} w_{m}=1 \text {, }
$$

$$
i_{m} w_{m} H_{m}=\sum w_{m} \eta_{m}=0,
$$

and

$$
\Sigma_{m} w_{m} \mu_{m}^{2}=\Sigma_{m} w_{m} \eta_{m}^{2}=1 / 3 .
$$

These are extended to includz also the component $\xi$ in the case of $(x, y, z)$ geometry. The last of the condftions [Eq. (2.27)] is required to get consistent balance and net flux equations, which in this case are obtained by sunmations over $m$ in Eq. (2.17) after muitiplying by the factors $1,3 \mu_{m}$, and $3 n_{m}$, respectively.

The finite cell formulation results from the discretization of position $(x, y)$ in the discrete ordinates equations. Again, the physical principles of Eq. (2.1) are retained. The finlte cell equation 1s vritten

$$
\begin{aligned}
& w_{m} A_{1, j}\left(N_{m, 1+1, j}-N_{m, 1-\frac{1}{2}, j}\right) \\
& +n_{m} B_{1, j}\left(N_{m, 1, j+\frac{1}{2}}-N_{m, 1, j-\frac{1}{2}}\right) \\
& +\sigma_{1, j} v_{1, j} N_{m, 1, j}=v_{1, j} s_{1, j},
\end{aligned}
$$

where the cell is defined by the coordinates $x_{1-y_{2}}$, $x_{1+y_{\xi}}, y_{j-\xi_{2}}$, and $y_{j+k_{2}}$, and the area and volume eleuents hy

$$
\begin{aligned}
& A_{1+\frac{1}{1, j}}=A_{1-\frac{1}{2}, j}=A_{1, j}=\Delta y_{j}, \\
& \text { and } \\
& B_{1, j+\frac{1}{2}}=B_{1, j-\frac{1}{2}}=B_{1, j}=\Delta x_{1}, \\
& v_{1, j}=\Delta x_{1} \Delta y_{j}=\left(x_{1+\frac{1}{1}}-x_{1-\frac{1}{2}}\right)\left(y_{j+\frac{1}{2}}-y_{j-\frac{1}{2}}\right) .
\end{aligned}
$$

In general, area elements divided by volume elements (here $A / V$ and $B / V$ ) have units of inverse length, that 1s, the unts of $\sigma$. Note that here, for a regular rectangular mesh, $A_{i, j}$ depends only in the $y$ Index $f$ and $B_{1, j}$ only on the $x$ index 1 . In conventicnal, siuplified nctation, omiting all central subscripts, Eq. $(2.28)$ becomes

$$
\begin{aligned}
& \mu A\left(N_{1+\frac{1}{2}}-N_{1-\frac{1}{2}}\right)+\eta B\left(N_{j+\frac{1}{2}}-N_{j-\frac{1}{2}}\right) \\
& +\sigma V N=V s .
\end{aligned}
$$

If the variable $z$ is present, a flux difference with 
the factor $\xi C$ is added. In this case $A=\Delta y \Delta z$, $\mathrm{g}=\Delta \mathrm{x} \Delta \mathrm{z}, \mathrm{c}=\Delta \mathrm{x} \Delta \mathrm{y}$, and $\mathrm{V}=\Delta \mathrm{x} \Delta \mathrm{y} \Delta \mathrm{z}$. If the time variable is present, a flux difference with a factor $v / v \Delta t$ is added.

Equation (2.28) is to be solved numarically for the central flux $x_{g, m, f, f}$ and the extrapolations to the cell boundaries which. In the case of $\mathrm{s}_{\mathrm{m}}$ and ${ }_{\mathrm{m}}$

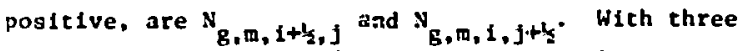
unknowns involved, Eq. (2.2.8) must be supplemented by two auxillary equations, that is, by difference schemes. The source cerms depend or the $N_{B, m, ~}, J$ functions but usually not strungly, therefore lterative means of solution can be used effectively. In other words, in any iterative step, the cerm $v_{1, j}$ $s_{i, j}$ in Eq. (2.28) is assured to be known. Equation (2.28) is taken to be exact in the sense that $\sigma_{1, j}, \sigma_{1, j} N_{m, 1, j}$, and $s_{1, j}$ represent approprtate volume averages of $0, \sigma x$, and $S$, and che remaining $\mathrm{x}^{\prime}$ 's similarly represent approprizte surface averages. Equations (2.28) and (2.32) clear1) reveal the conservation nature of the transpost equation. The tern $|\mu| A N_{i+\frac{1}{3}}$ signifies, for example, the number of particles leaving (entering if $u<0$ ) the cell over the face defined by the Index pair $\left(1+\frac{1}{y}, f\right) ;|n| B N_{f-\frac{1}{2}}$ the number entering (leaving if $\eta>0)$ the cell over the face $\left(1, j-\frac{k}{2}\right)$; and $v(S-\sigma N)$ the net change of particles th the cell from emissions and renovals. Here the source term Vs includes in-scattering from its uwn and other groups, the collision cerm von losses in the form of out-scatterIng to its own or other groups.

The finite cell equation for the characteristic formulation may be writteri

$$
T\left(N_{h+\frac{1}{2}}-N_{h-\frac{1}{2}}\right)+V w=v s,
$$

where the effective Input or output area, or target area, is defined by

$$
T=|\mu| A+\ln \mid B
$$

and where $\mathrm{TN}_{h+\frac{1}{2}}$ and $\mathrm{TN}_{h-\frac{1}{2}}$ are given by

$$
\mathrm{TN}_{n+\frac{1}{2}}=|\mu| \mathrm{AN}_{1+\frac{1}{2}}+|n| \mathrm{BN}_{j+\frac{1}{2}}
$$

and

$$
\mathrm{TN}_{h-\frac{1}{2}}=|\mu| \mathrm{AN}_{1-\frac{1}{2}}+\ln \mid \mathrm{BN}_{j-\frac{1}{2}} .
$$

These two formulas are valid for the princidal octant. In the following text, most formulas will only be developed for that octant. The sign and index rules by octant are given In Table 11-1.

Equation (2.33), the one-dimensionalized forti of Eq. (2.32), is readily solved hy the methods described in the next section for infinite plane $(x)$ geometry. The solution is penerated in a prescribed sequence, west ceil by mesh cell, for each discrete direction and for each velosity proup. To control round-off errors in the calculactons, the direction of evaluation through the mesh must be consistent with $a_{m}$. Therefore, if $\mu_{m}$ is negative and positive. the evaluation progresses in the negative $x-$ direition and the positive $y$-dfrection. The process assumes an estimate for the source term, as it begins. tive uydating of the source whenevar practical. as it continues, and the recaiculation of the source, as it terminates, chereby setefing up an iterative loop. In many problems, the calculations entall two or more iterative loops, usually nested, in which the number of cycles is limited by presiribed convergence criteria. The process also assumes known Inputs from adjolning cells, prevlous in the calculating sequence, or, In exceptional cases, from boundary conditions. As a result, Eq. (2.33) is an equation in two unknowns, $y$ and $\mathrm{N}_{h+l_{2}}$. But, as stated above, solution is made posible by introducing a difference schere.

As indlcated. the solution of Eq. (2.33) appears as a pais of positive numbers, one for the midcell flux $N$ and the other fer the extrapolated flux $x_{h+l}$. $A$ weight $P$ is assoclated with the pair, where $P=1$ In the nurmal, smell cell case, and a $P$ subject to $C \leq P<1$ is determined in the large cell case. The solution, as explained further in Sec. III, depends

\begin{tabular}{|c|c|c|c|}
\hline $\begin{array}{l}\text { Oetant } \\
\text { Number }\end{array}$ & $\begin{array}{l}\text { Stga } \\
\text { of } \mu\end{array}$ & $\begin{array}{l}\text { S1gn } \\
\text { of } n\end{array}$ & Indices in the Terms of \\
\hline 1 & + & + & $1+\frac{1}{2}, j+\frac{1}{2}$ \\
\hline 2 & - & + & $1-\frac{1}{2}, j+\frac{1}{2}$ \\
\hline 3 & + & - & $1+x_{2}, j-\frac{x}{2}$ \\
\hline 4 & - & - & $\pm-\frac{x}{2}, j-\frac{x}{2}$ \\
\hline
\end{tabular}
on the difference relation

TABLE II- 1 SIGN AND INDEX RULES FOR $(x, y)$ GEOMETRY 


$$
s_{h+\frac{1}{2}}-N_{h-s_{2}}=(1+P)\left(N-N_{h-\frac{1}{2}}\right) \text {, }
$$

and is found to be

$$
N=\frac{(1+P) T N_{h-\frac{1}{2}}+V S}{(1+P) T+O V}
$$

together with

$$
N_{h+\frac{1}{2}}=\frac{[(1+P) T-P V \sigma] N_{h-\frac{1}{g}}+(1+P) V S}{(1+P) T+\sigma V} .
$$

The difference scheme [Eq. (2.37)] implies an $S_{n}$-type approximation to the flux for $P=1$, that is, an approximation by means of connected straight-ine segments. In the exceptional case, $0 \leq P<1$, the line segments are allowed to be discontinuous. The $P=0$ case is referred to as the step scheme and the $0<P<1$ case is referred to as tive sloping step scheme. Equation (2.37) with $P=1$ implies the diamond difference scheme, accurate to second-order terms in ís.

It remains to determine the individual extrapolations, or average outputs, knowing the combined average output $\mathrm{N}_{h+l}$. If the direction lies in the principal octan:, these ousputs are $N_{i+\zeta}$ and $N_{j+l_{2}}$. Several methods will be discussed.

In the diamond difference schene, $\mathrm{N}_{1+\frac{1}{2}}$ and $\mathrm{N}_{\mathrm{j}+t_{\mathrm{g}}}$ are obtained from the following detalled schemes.

$$
N_{1+\frac{1}{2}}=(1+P) N-P N_{1-1 / \frac{1}{2}}
$$

and

$$
N_{j+\frac{1}{2}}=(1+P) N-P N_{j-\frac{1}{2}}
$$

which are consistent with Eq. (2.37). This is readily seen by combining the two equations with the weights $|M| A$ and $|\eta| B$, respectively, which yields

$$
\mathrm{TN}_{h+\frac{1}{2}}=\mathrm{T}(1+\mathrm{P}) \mathrm{N}-\mathrm{TPN}_{\mathrm{h}-\frac{\mathrm{K}}{2}}
$$

In some situations, Eqs, (2.40) and (2.41) may produce one negative extrapolation. This is referred to as flux skewing, and positivity of the errant extrapolation may be restored by a skewing correction as follows. The correction is formulated so that $\mathrm{N}_{\mathrm{h}+\frac{1}{2}}$ is left unchanged. The set of extrapnlations is revieed each time a particular one is found to be negative. The following simple skewing correction, satisfying the condition, may be used. If $\mathrm{N}_{i+\frac{1}{2}}$ is cested and found to be negative, $N_{1+\frac{1}{2}}$ is set to zero after first computing a factor $f$.

$$
f=-x_{i+\frac{1}{2}} /\left(w_{h+\frac{1}{2}}-w_{i+\frac{1}{2}}\right) .
$$

The set of extrapolations is then modified by

$$
N_{\ell+\frac{1}{2}}+f\left(N_{h+\frac{1}{2}}-N_{\ell+\frac{1}{2}}\right)-N_{l+\frac{1}{2}}
$$

where $\ell$ denotes the sequence of Indices, here 1 and $j$. The calculation for $\ell=1$ can of course be omitted because in this case the ravised $\mathrm{N}_{1+l_{2}}$ equals zero by construction. If $\mathrm{N}_{i+j_{2}}$ is not negative, one goes on to test $N_{j+\frac{1}{2}}$, etc. In the $(x, y)$ case, if $\mathrm{N}_{i+\frac{1}{2}}<0, \mathrm{~N}_{\mathrm{j}+l_{2}}$ need not be tested because at least one of the outputs must be positive.

The extrapolations may be based on the idea that one is dealing with a single strean and that therefore the coordinate directions are auxiliary, less important directions. This is the characteristic approach in which Eq. $(2.33)$, not Eq. (2.32), is the basic equation. The simplest extrapolation of this type is based on

$$
x_{i+x_{2}}=N_{j+x_{2}}=N_{h+x_{2}}
$$

This rather crude scheme implies a sloping step scheme in the coordinate directions and it reiuires no skewing correctiono. A more refined method is based on interpolation uعing $N$ and $N_{h+1 / 2}$ and the projections of the output points on the line through the $N$ and $N_{h+l}$ points. This leads to

$$
N_{i+\frac{1}{2}}=N+D|u| \Delta x\left(N_{h+i_{2}}-N\right)
$$

and

$$
N_{j+\frac{1}{2}}=N+D|n| d y\left(N_{h+\frac{1}{2}}-N\right)
$$

with $\mathrm{D}$ to be determined so that the correct combined output $\mathrm{TW}_{\mathrm{h}+\frac{\mathrm{t}}{2}}$ is obtained. One finds

$$
D=T /\left(\mu^{2}+n^{2}\right) v \text {. }
$$

Finally, the most refined characteristic scheme is 
in this geometry equivalent to che diamond scheme.

In this and the previous case, it becomes necessary to test for skewing corrections.

The extrapolation methods based on Eq. (2.45) or Eqs. (2.46) and (2.47) exercise constderable restraint on the variation of the angular flux $N$. Th/sy may therefore be regarded as methods for smoothing the flux and may, together with the diamond scheme, be combined with a smoothing of the inputs by one of the methods described in Ref. 1, Sec. V. In general, smoothing methods can bring about considerable mitigation of ray effects. However, from all indications, the effect of smoothing extends over rather few intervals. Therefore, smoothing is not likely to be a complete remedy for ray effects.

The discrete $s_{n}$ method, based on solving Eq. (2.32) or $E c_{2}$. (2.33) by means of the diamond scheme, performs rather well over a wide range of applications. Actually, in some instances it performs too well -- 500 much in accordance with the assunptions made, particularly to the discrete ordinates approximation. In that basic, rather crude approximation, isotropic sources are replaced by spoke-like sources with fairly sparse spokes. In many probiems this does not matter because scattering and other natural smoothing processes are present. In some problems, however, the natural smoothing is not sufficient in magnitude or uniform distribution over the system. In such cases, wavelike distortions in the flux may beccme evident. This is referred to as ray effects. It is a form of extraneous behavior, an imprint on $N$ of the discrete representation for $\Omega$. Ray effects become possible when two or more time and position variables are present.

Several methods lave been propozed for major reduction or elimination of ray effects. A few, rather elaborate procedures have proved to be effective. However, these have also proved to be costly in computer time and storage and to slow down the convergence of 1 terative sequences. The most direct means of alleviating ray effects is of course to increase the order $n$ of approximation. Unfortunately, this is an Ineffective method because a very large $n$ is required.

A simplifled method, which probably has some adverse effect on convergence but demands little in terms of added computer time and storage, is based on a modified form of the transport equation, iike 5q. (2.16). Here, for $n=2$, a single excess tern. $15 \mu n Q$, is excluded from the derivative teras. In the general case, fluxes are obtainea for $n(n+2) / 2$ directions which can be used to determine a polynomfal in $\mu$ and $n$, complete to degree $n-1$, plus a few terms of degree $n$. The complete part requires $n(n+1) / 2$ fluxes, leaving to terms to determine as many excess terms. Ir the simplified method, as ar. aporoximation, one sets the excess terms equal to $15 c_{n} \mu \Pi Q$ where $c_{2}=1.0$, and $c_{n}, n>2$, is determined by solving some basic protlem for a few $n$ 's. say $n=4,6,8$, and 12 . The quantities $c_{n}$ would be selected to give as smooth a solution as possible. A suitable problem might be a centrally located source in a vacuous and square $(x, y)$ configuration. Assuming that such $c_{n}$ can be determined, the regular difference procedure with $P=1$ leads to a source correction $\Delta S$ of the form

$$
\left.\Delta S=30 c_{n} \mu n !|\mu| A+\ln \mid B\right) Q .
$$

Mora explicitly, the vS source terms in sqs. (2.32) and (2.33) are replaced by $V(S+\Delta S)$.

The mitigation of ray effects has two main results, one highly desfrable, the other undesirable but usually acceptable. The desirable result is that the scalar flux $\overline{\mathrm{N}}$ becomes woother and more realistic. The undesirable result is that the angular flux generally becomes less smooth.

\section{SOLUTION IN THE ONE-DIMENSIUNAI PLANE CASE}

In infinite plane $(x, \mu)$ geometry the differential equation for particle transport is given by

$$
\mu \frac{\partial}{\partial x} N(x, \mu)+\sigma(x) N(x, \mu)=s(x) .
$$

This assumes isotropic emissions and interactions, so that $S$ and $\sigma$ are Independent of $\mu$, and a single component $\mu$ of $\Omega,-1 \leq \mu \leq 1$, representing direction relative to the space coordinate $x$.

In the discrete ordinates formulation of the above, with ordinates $\mathrm{N}_{\mathrm{bil}}$ defined by

$$
N_{m}(x)=N\left(x, \mu_{m}\right),
$$

Eq. (3.1) is replaced by a system of equations.

$$
\mu_{m} \frac{\partial}{\partial x} N_{m}(x)+\sigma(x) N_{m}(x)=s(x) .
$$


Here a $u$-interval of length. $\Delta u_{m}=u_{m+\frac{1}{2}}-\mu_{m-\frac{1}{2}}$, or welght $w_{m}=1 / 2 \mu_{m}$, is assigned to each distinct $\dot{m}_{m}$, $\mathrm{n}=1,2, \ldots, \mathrm{n}$, where $\mathrm{n}$ is the order of angular approximation, $n \neq 2,4, \ldots$, In general, a quadrature set $\left(w_{i n} \cdot H_{m}\right.$ ) for Eq. (3.3) must sat isfy the fallowing conditions.

$$
\begin{aligned}
& \Sigma_{m} w_{m}=1, \\
& \Sigma_{m} w_{m} w_{m}=0,
\end{aligned}
$$

and

$$
\sum_{m m} w_{m}^{2}=1 / 3
$$

The abscissas $\mu_{t \mathrm{n}}$ and related $u$ neighborhoods are normally placed symecrically with respect to $u=0$ on the interval $(-1,1)$, an arrangement that implies Eq. (3.5). The suigjert of quadrature is discussed in more detail in sec. $v 1$.

The finite difference equation corresponding to Eq. (3.3) is written

$$
\begin{aligned}
& 1_{m}\left(A_{i+\frac{1}{2}} N_{m, 1+\frac{1}{2}}-A_{t-\frac{1}{2}} N_{m, 1-\frac{1}{2}}\right) \\
& +\sigma_{i} v_{i} N_{m, i}=v_{i} s_{1},
\end{aligned}
$$

where, in plane geometry,

$$
A_{i+\frac{1}{2}}=A_{i-\frac{1}{2}}=A_{1}=1
$$

and

$$
v_{1}=\Delta x_{i}=x_{1+\frac{1}{2}}-x_{1-\frac{1}{2}}
$$

with $A_{i}$ defined by

$$
A_{i}=\frac{1}{2}\left(A_{1+\frac{1}{2}}+A_{i-\frac{1}{2}}\right)
$$

In Eq. (3.7), $s_{i}, N_{m, i}$, and $\sigma_{i} N_{m, i}$ represent volume averages of $S(x), N_{m}(x)$, and $\sigma(x) N_{m}(x)$ over $\left(x_{1-\frac{1}{2}}, x_{i+\frac{1}{2}}\right)$. In many situations $\sigma$ is constant within intervals so that $\sigma(x)=\sigma_{i}$. Physically, $v_{i} s_{i}$ and $\sigma_{1} v_{i} N_{m, i}$ represent total releases and removals of particles, respectively.

Using the above formulas for area and volume elements, and the convention of omitting central subscripts, Eq. (3.7) becomes

$$
\mu\left(N_{i+\frac{1}{2}}-N_{i-\frac{1}{2}}\right)+\sigma \Delta x N=\Delta x S
$$

In the general context of the method of characteristics, Eq. (3.11) is written

$$
\left(N_{h+\frac{1}{2}}-N_{h-h / 2}\right)+u N=u S / \sigma
$$

where $u=\sigma V / T$ and, for the present geometry,

$$
u=\sigma v / T=\sigma_{h}\left\langle x_{h} / u_{m} !\right.
$$

Here $h$ is defined so that $x_{h-\frac{1}{2}}$ is the boundary of intiow and $x_{h+l}$ is the boundary of out?low. Therefore

$$
v_{h+\frac{1}{2}}-s_{h-\frac{1}{2}}= \begin{cases}N_{m, i+\frac{1}{2}}-s_{m, i-\frac{1}{2}}, & u_{m} \text { positive, } \\ x_{m, 1-\frac{1}{2}}-s_{m, i+\frac{1}{2}}, & i_{m} \text { negative. }\end{cases}
$$

The thickness of a given mesh cell $V / T$ is obtained by dividing the volume element $V$ by the effective target area $A$. (V/T)S replaces $u S / \sigma$ in Eq. (3.i2) if $\sigma=\mathbf{u}=0$.

Becduse $\sigma$ often varies a great deal, and irregularly, with particle velacity and material composirfon (hence with position in space), it is frequently timpossible to construct a mesh so that $u$ is sufficiently small everywhere for all velocity groups and all beam directions. Therefore, in findIng numerical solutions, one must be prepared for large as well as small u dimensicns.

The source uS/O and sink uN terms in Eq. (3.12) can clearly be decreased (or increased) by the same amount with no real change to the equation, which points to an ambigulty in the transport equation. The magnitude of this depends in general on $x$ and $u$, may vary irregularly, and may be difficult to detine in any precise manner. However, when present, the ambiguity can be used to advantage to decrease $u$, by decreasing uN, to obtain an effective optical thickness $u^{\prime}$. If one permits a negative $u^{\prime}$ but not a negative modified source, uN can be decreased by as much as us/o. Performing line maximum subtraction, one obtains

$$
N_{h+\frac{1}{2}}-N_{h-\frac{1}{2}}+u \frac{N-S / O}{N} N=0
$$

as a replacement for Eq. (3.12). On this basis, and with some means for estimating $N, u$ 'may be defined by 


$$
u^{\prime}=u^{N-S / O}
$$

Further, u' may be written

$$
u^{\prime}=\sigma^{\prime} \mathrm{V} / \mathrm{T},
$$

thus defining $\sigma^{\prime}$, the effective removal probability. Two derived functions, the scalar flux $\bar{N}$ and the current or net flux I, are of particular importance in transport theory. They are defined by

$$
\bar{N}(x)=\frac{1}{2} \int_{-1}^{1} N(x, \mu) d \mu
$$

and

$$
I(x)=\frac{1}{2} \int_{-1}^{l} \mathrm{~N}(\mathrm{x}, \mu) \mu \mathrm{d} \mu .
$$

Associated with these functionals are two derived equations, the balance equation and the net flux equation, given by

$$
\frac{\partial}{\partial x} I(x)+\sigma(x) \bar{N}(x)=S(x)
$$

and

$$
\frac{\partial}{\partial x} \bar{N}(x)+3 \sigma(x) I(x)=0 .
$$

The derivation is by integration of Eq. (3.1) over 14 frum -1 to 1 after multiplying the terms by $\frac{1}{2} \mathrm{~d} \mu$ and $\frac{3}{2} \mu d \mu$, respectively, and by using, in the second integration, the diffusion approximation to $N(x, \mu)$.

$$
N(x, \mu)=\bar{N}(x)+3 \mu I(x)
$$

In the finite difference case, the corresponding functionals and equations, consistent with the analytical formulas in the limit of small $\Delta x$ and $\Delta \mu$, are

$$
\begin{aligned}
& \bar{N}_{1}=\sum_{m} w_{m} N_{m}, \\
& I_{1}=\sum_{m} w_{m} H_{m} N_{m, 1}, \\
& I_{1+\frac{l}{2}}-I_{1-\frac{1}{2}}+\sigma_{1} \Delta x_{1} \bar{N}_{1}=\Delta x_{1} S_{1},
\end{aligned}
$$

$$
\bar{N}_{i+1}-\bar{N}_{1-\frac{1}{2}}+3 \sigma_{i} \Delta x_{1} I_{i}=0
$$

with the approximation

$$
N_{m, 1}=\bar{N}_{1}+3 \mu_{m} I_{1}
$$

used with Eq. (3.26).

Physically, the flux functions $\mathrm{N}_{\mathrm{m}}, \bar{N}$, and $I$ should be continuous in $x$, also in $\mu$ with some exceptions. In the numerical work, $\mathrm{N}_{\mathrm{m}}$ is sometimes made discontinuous at a cell boundary just after the particles it represents have crossed the boundary. Nevertheless, continuity of $\bar{N}$ and I can be maintained if, in forming $\vec{N}$ and $I$ at a boundary, values of $N_{m}$ just before (or at) the boundary are used. The consistency of the above finite difference equations with their analytical counterparts depends, in the first place, on making sure that $\bar{N}$ and $I$ are continuous and, in the second place, on the quadrature conditions [Eqs. (3.4) through (3.6)].

The consistency of the analytical and finte difference formulations is important for the proper convargence of the solutions with $n$, the order of directional approximation, and for obtaining high accuracy for relatively low $n$, say for $n=4,6$, and 8. The second consideration is important because convergence as $n$ is increased tends to be slow. As seen above, consistency for plane geometry is readily established. For spherical and cylindrical geometry, however, consistency is somewhat more difficult to achleve and has a definte effect on the method of numerical solution.

Equation (3.12) is solved numerically by using the weighted dtamond scheme, a simple difference scheme, given by

$$
N_{h+1}=(1+P) N-P N_{h-\frac{1}{2}}
$$

which may also be written

$$
N_{h+l_{2}}-N_{h-\frac{1}{2}}=(1+p)\left(N-N_{h-\frac{1}{2}}\right),
$$

where $\mathrm{P}$ is a parameter, $0 \leq \mathrm{P} \leq 1$. The auxiliary relation [Eq. (3.28)], together with the main equation [Eq. (3.12)], gives two equations for obtaining the two unknowns: the midcell flux $\mathrm{N}$ and the extrapolated flux $\mathrm{N}_{\mathrm{h}+\mathrm{l}_{2}}$. In the modified diamond scheme (MDS), $P$ is set to unity for small intervals and 
computed from a given formula for large intervals. The use of $P$ - $I$ (the DS scheme) assumes that $N$ is linear in $x$ in the interval $\left(x_{1-\frac{1}{2}}, x_{1+l_{2}}\right)$. Physical$1 y, p=1$ means that the situation may be regarded as elliptic, or mildly or moderately hyperbolic and $P<1$ means that the situation is decisively hyperbolic.

The weighted dlamond scheme, also called the sloping step scheme, may be given the interpretation 1llustrated in Fig. 3.1. One finds $\mathrm{N}_{\mathrm{h}-\mathrm{k}_{2}}^{+}$, the pastcrossing value of $\mathrm{N}_{h-\frac{h}{2}}$, to be

$$
N_{h-\frac{1}{2}}^{\prime}=2 N_{h}-N_{h+\frac{1}{2}}=(1-P) N_{h}+P N_{h-\frac{1}{2}}
$$

and, for the discontinuicy $N_{h-\frac{1}{2}}-N_{h-\frac{1}{2}}^{\prime}$ at $x_{h-\frac{1}{2}}$

$$
N_{h-\frac{1}{2}}^{\prime}-N_{h-\frac{1}{2}}=(J-P)\left(N_{h}-N_{h-\frac{1}{2}}\right) .
$$

Note that continuity of $N$ requires that $P$ be unity.

Substituting Eq. (3.28) with $\mathrm{P}=1$ in the diEference equation [Eq. (3.12)], and solving for $\mathrm{N}$, one oblains

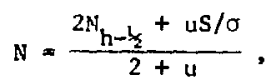

and, from this, the extrapolation

$$
N_{h+\frac{1}{2}}=2 N-N_{h-\frac{1}{2}}=\frac{(2-u) N_{h-\frac{1}{2}}+2 u S / \sigma}{2+u} .
$$

For the general case of arbitrary $P$, one finds

$$
N=\frac{(1+P) N_{h-\frac{1}{2}}+u S / \sigma}{1+P+U}
$$

and

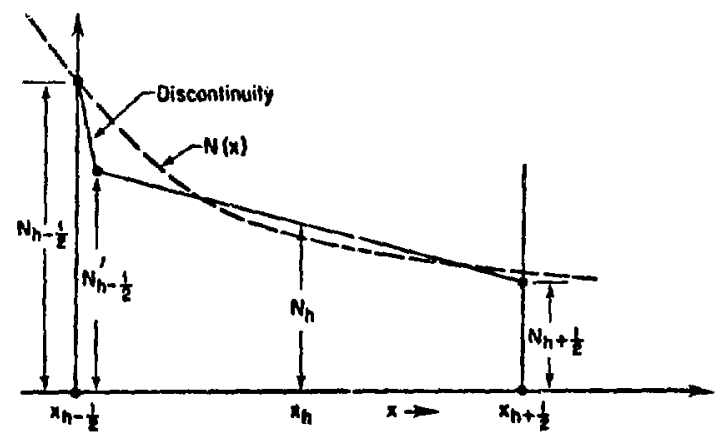

Fig. 3.1. Interpretation of difference schenc.

$$
\begin{aligned}
N_{h+\frac{1}{2}} & =(1+P) N-P N_{h-\frac{1}{2}} \\
& =\frac{(1+P-u P) N_{h-\frac{1}{2}}+(1+P) u S / 0}{1+P+u} .
\end{aligned}
$$

Here, one notes first that Eqs. (3.32) chrough (3.35) represent weighted averages of $\mathrm{N}_{h-1}$ and $\mathrm{s} / \sigma$ with weights that sum to unity. Second, one notes that the coefficient of $\mathrm{N}_{\mathrm{h}-\mathrm{t}}$ in Eq. (3.35), which analytically should equal the penetration probability $e^{-\mu}$, is an approximation to $e^{-u}$. Expanding for small $u$ one obtains

$$
\begin{aligned}
& \frac{1+P-u P}{1+P+u}=1-\frac{u}{1+\frac{u}{1+P}} \\
& =1-u+\frac{u^{2}}{1+P}-\frac{u^{3}}{(1+P)^{2}}+\cdots .
\end{aligned}
$$

This is accurate to second-order terms for all $P$ and to third-order terms fot $P=1$. The ratio is clearly not a good approximation to $e^{-u}$ if $u$ is so large that it becomes negative, that is, if $\mathrm{u}>(\mathrm{I}+\mathrm{P}) / \mathrm{P}$ or $\mathrm{P}>1 /(\mathrm{u}-1)$.

Further, from the numerlcal solutions, one observes that the sequence of fluxes, $N_{h-\frac{1}{2}}, N_{h}, N_{h+\frac{1}{2}}$, is monotonely increasing if $\mathrm{S} / \sigma>\mathrm{N}_{\mathrm{h}-\mathrm{t}_{2}}$ (the growth case), and nonincreasing if $s / \sigma \leq \mathbb{N}_{h \cdot t}$ (the decay case). Also, by using Eq. (3.34), Eq. (3.31) for the discontinuity can be written

$$
N_{h-\frac{1}{2}}-N_{h-\frac{1}{2}}=\frac{u(1-P)\left(S / \sigma-N_{h-\frac{1}{2}}\right)}{1+P+u},
$$

which shows (if $P<1$ ) that the discontinuity at $x=x_{h-\frac{1}{2}}$ is positive in the growth case and negative or zero in the decay case. Using the diamond solution (DS) for $N$, one deduces a second form for u'.

$$
u^{\prime}=u \frac{N-S / O}{N}=\frac{N_{h-\frac{1}{2}}-N}{\frac{1}{2} N} \text {, }
$$

and, from these equalities,

$$
\left(u-u^{\prime}\right) N=u S / \sigma
$$

and

$$
N_{h-\frac{1}{2}}=\left(1+1+2 u^{\prime}\right) N .
$$

Hence. In terms of $N_{h-1 / 2}$ and $u^{\prime}$, 


$$
N=\frac{N_{h-\frac{1}{2}}}{1+\frac{1}{2} u^{1}} \text {, }
$$

and

$$
N_{h+\frac{1}{2}}=\frac{\left(1-\frac{1}{2} u^{\prime}\right) N_{h-b_{2}}}{1+\frac{1}{2} u^{\prime}}=\left(1-\frac{1}{2} u^{\prime}\right) N .
$$

The weighted diamond scheme has two primary virtues. Fi.st, if is arithmetically simple, especially for $P=1$, and is therefore economical with computer time. Second, it is invariant with respect to the source/sink ambi: ity in the transport equation. This makes it ry accurace, bastcally to second-order terms in $u$ ' rather than in $u$ as one would first expect. The invariance of the scheme is established in Theorem 3.1 below. Note that $u^{\prime}$ is often substantially smaller than $u$ and may, in high-scattering situations, be smaller by one or more orders of magnitude.

The modfied diamond scheme (MDS) can now be more precisely defined. One first computes values fur $N$ and $N_{h+1}$ from Eqs. (3.32) and (3.33) based on $P=1$ and then $u^{\prime}$ from Eq. (3.38). These values constitute the final solution for the interval unless $u^{\prime}$ is greater than $u_{0}^{\prime}$, where $u_{0}^{\prime}$ is a given parameter, $u_{0}^{\prime} \sim 1$. If $u^{\prime}>u_{0}^{\prime}$, which presumably would be infrequent, $N$ and $N_{h+\frac{1}{2}}$ are recalculated using Eqs. (3.34) and (3.35) and a given formula for $P, P=P\left(u^{\prime}\right)$. Continulty of $P\left(u^{\prime}\right)$ at $u^{\prime}=y_{0}^{\prime}$ is assumed so that $P\left(u_{0}^{i}\right)=1$. A simplified version of the MDS method, the limit version, will be defined and discussed later.

Theorem 3.1. The solution of Eq. (3.12) by the weighted diamond scheme, using a constant $P$, is independent of the source/sink ambiguity in the equation, that is, of any equal reduction in the source and sink terms.

Here, assuming a reduction of the source term by quS/o and an lavarlant solution, one wants to show that the sink term is also reduced by quS/ $\sigma$. From Eq. (3.34), without relustion, one has

$$
(1+P+u)\left(N-N_{h-\frac{1}{2}}\right)=u\left(S / \sigma-N_{h-\frac{1}{2}}\right) .
$$

With the source reduced and $u$ replaced by : ', assumIng the same solution for $\mathrm{N}$, one has

$$
\begin{aligned}
& \left(1+P+u^{\prime}\right)\left(N-N_{h-\frac{1}{2}}\right) \\
& =u^{\prime}\left[(1-q) S / \sigma^{\prime}-N_{h-\frac{1}{2}}\right] .
\end{aligned}
$$

Subtraction of Eq. (3.44) from Eq. (3.43), noting that $u / \sigma=u^{\prime} / \sigma^{\prime}$, gives

$$
\left(u-u^{\prime}\right)\left(N-N_{h-\frac{1}{2}}\right)=q u S / \sigma-\left(u-u^{\prime}\right) N_{h-\frac{1}{2}}
$$

Consequently,

$$
u^{\prime} \mathbf{N}=\mathbf{u}(\mathrm{N}-\mathrm{qS} / \sigma),
$$

which shows that un is also reduced by quS/ $\sigma$.

Theorem 3.1 provides a reasonable basis for using Eqs. (3.16) and (3.38) to estimate the effective optical thickness $u^{\prime}$. The estimate appears to be on the low side. It does not seem worthwhile, however, to 1terate on $\mathrm{N}$.

Theorem 2.2 . If $P=P\left(u^{\prime}\right)$ is chosen so that $P=1$ for $u^{\prime} \leq 2$ and $P \leq 1 /\left(u^{\prime}-1\right)$ for $u^{\prime}>2$, then, on the normal assumption that $u, N_{h-\frac{1}{2}}$, and $u s / \sigma$ are nonnegative, $\mathrm{N}_{\mathrm{h}+\frac{\mathrm{t}}{2}}$ is also nonnegative.

Thts theorem is verified by first writing Eq. (3.35) as follows and by adding and subtracting equal terms.

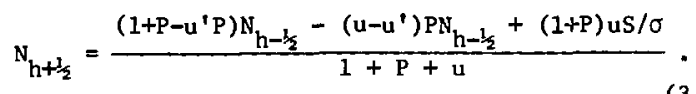

By application of Eqs. (3.39) and (3.40), this is simplified to

$$
N_{h+\frac{1}{2}}=\frac{\left(1+P-u^{\prime} P\right) N_{h-\frac{1}{2}}+\left(1-\frac{1}{2} u^{\prime} P\right) u S / \sigma}{1+P+u}
$$

Clearly, if $P=1$ and $u^{\prime} \leq 2, N_{h+\frac{1}{2}}$ is nonnegative. Also, if $P \leq 1 /\left(u^{*}-1\right)$, the coeffictent of $N_{h-\frac{1}{2}}$ is nonnegative. With this restriction on $P$, and with $u^{\prime}>2$, one can write

$$
1-13 u^{\prime} P \geq \frac{u^{\prime}-2}{u^{\prime}-1}>0 \text {. }
$$

Therefore, the coefficient of $S / \sigma$ is also nonnegative, which completes the proof. Note that if $P$ approaches $1 / u^{\prime}$ for very large $u^{\prime}, N_{h+\frac{1}{2}}$ approaches $\frac{1}{2} \mathrm{~s} / \sigma$. 
Theorem 3.3. If $P$ satisfles

$P \leq 1 / \mathbf{u}^{\prime}<1 /\left(\mathbf{u}^{\prime}-1\right)$, then, in the decay

case, $N_{h+h_{2}}$ is limited from below by $k_{2} 5 / \sigma$.

To prove this, one observes first, by using Eq. $(3.40)$, that

$$
\mathrm{N}_{h-\frac{1}{2}}=\left(1+\frac{1}{2 u^{\prime}}\right) \mathrm{N} 2\left(1+\frac{1}{2 u^{\prime}}\right) \mathrm{s} / 0 .
$$

After using this in Eq. (3.48) to put $\mathrm{N}_{\mathrm{h}-\frac{1}{2}}$ in terms of $\mathrm{S} / \sigma$ and decrease the numerator, it remains to demonstrate that

$$
\begin{aligned}
& \left(1+P-u^{\prime} P\right)\left(1+\frac{1}{2} u^{\prime}\right) \\
& +\left(1-\frac{1}{2} u^{\prime} P\right) u^{\prime} \geq \frac{1}{2}(1+P+u) .
\end{aligned}
$$

Solving this inequality for $P$, one obtains

$$
P \leq \frac{u+u^{\prime}+1}{u^{\prime}\left(u+u^{\prime}+1\right)-1}
$$

winich is satisfled by $P \leq 1 / U^{\prime}$.

It seems reasonable, in view of the above, to complete the MDS scheme by taking $u_{0}^{\prime}=1$ and using $P\left(u^{\prime}\right)=1 / u^{\prime} I \bar{I} u^{\prime}>u_{0}^{\prime}$ An acceptable generalization of this is to use

$$
P\left(u^{\prime}\right)=\frac{u^{\prime}+\left(1-2 u_{0}^{\prime}\right)}{\left(u^{\prime}\right)^{2}+\left(1-2 u_{0}^{\prime}\right) u^{\prime}+\left(u_{0}^{\prime}-1\right)^{2}},
$$

val1d for $0 \leq u_{0}^{\prime} \leq 1$ where $P\left(u_{0}^{\prime}\right)=1$ and

$$
P^{\prime}\left(u^{\prime}\right)_{u^{\prime}=u_{o}^{\prime}}=0
$$

$u_{0}^{\prime}<1$. This reduces to $P=1 / u^{\prime}$ for $u_{0}^{\prime}=1$ and to

$$
P\left(u^{\prime}\right)=u^{\prime} /\left[\left(u^{*}\right)^{2}+1 / 4\right]
$$

for $u_{0}^{1}=1 / 2$. Table III-1 gives some comparisons for $P$.

Many other possibllities exist for choosing $P\left(u^{\prime}\right)$ for $u^{\prime}>u_{o}^{\prime}$. For example,

$$
P\left(u^{\prime}\right)=\frac{u^{\prime}+2\left(1-u_{0}^{\prime}\right)}{\left(u^{\prime}\right)^{2}+\left(1-2 u_{0}^{\prime}\right) u^{\prime}+\left(u_{0}^{\prime}-1\right)^{2}+1} \text {, }
$$

$0 \leq u_{0}^{\prime} \leq 2$, with $P\left(u_{0}^{\prime}\right)=1$ and $\mathrm{Eq}$. (3.54) is satisfled for $u_{0}^{\prime}<2$. For $u_{0}^{\prime}=2,1, \frac{1}{2}$, Eq. $(3.56)$ gives

$$
P\left(u^{\prime}\right)=1\left(/\left(u^{\prime}-1\right)\right.
$$

$$
P\left(u^{\prime}\right)=u^{\prime} /\left[\left(u^{\prime}\right)^{2}-u^{\prime}+1\right]
$$

\begin{tabular}{|c|c|c|c|c|}
\hline$\underline{\mathbf{u}}$ & $\underline{1}(u-1)$ & $\underline{w} /\left(u^{2}+1 / 4\right)$ & $\underline{1 / u}$ & Eq. $(3.63)$ \\
\hline 0.5 & & 1.0000 & & 0.8467 \\
\hline 1.0 & & 0.8000 & 1.0000 & 0.7183 \\
\hline 1.5 & & 0.6000 & 0.6667 & 0.6115 \\
\hline 2.0 & 1.0000 & 0.4706 & 0.5000 & 0.5232 \\
\hline 2.5 & 0.6667 & 0.3846 & 0.4000 & 0.4505 \\
\hline 3.0 & 0.5000 & 0.3243 & 0.3333 & 0.3907 \\
\hline 4.0 & 0.3333 & 0.2462 & 0.2500 & 0.3010 \\
\hline 5.0 & 0.2500 & 0.1980 & 0.2000 & 0.2395 \\
\hline
\end{tabular}

and

$$
P\left(u^{\prime}\right)=\left(u^{\prime}+1\right) /\left[\left(u^{\prime}\right)^{2}+1.25\right] \text {, }
$$

respectively. It is not clear he:e if, for some siificiently small $u_{0}$, the relation $\mathrm{N}_{h+\frac{1}{2}} \geq 1_{2} \mathrm{~S} / \sigma$ holds. Assuming a constant source $S$ ovex the Interval $\left(x_{h-\frac{1}{2}}, x_{h+\frac{1}{2}}\right)$, one can derive an analytical expression

TABLE III-1

VALUES OF P FROM FORMULAS AS GIVEN 
for $\mathrm{N}_{\mathrm{h}+\frac{1}{2}}$ by integration, obtaining the total penetration

$$
N_{h+\frac{1}{2}}=N_{h-\frac{1}{2}} e^{-U}+\left(1-e^{-u}\right) s / \sigma,
$$

where $u=\sigma V / T$. Substituting this in Eq. (3.12), an analytical expression for $\mathrm{N}$ is found.

$$
\begin{aligned}
N & =N_{h-\frac{1}{2}}\left(1-e^{-u / u}\right) \\
& +\left[1-\left(1-e^{-u} / u\right] s / \sigma\right.
\end{aligned}
$$

Here, as in the case of Eqs. (3.34) and (3.35), one observes that $\mathrm{N}$ and $\mathrm{N}_{\mathrm{h}+\frac{1}{2}}$ are weighted averages of $\mathrm{N}_{\mathrm{h}-\frac{\mathrm{l}}{2}}$ and $\mathrm{s} / \sigma$. One also notes that $\mathrm{N}$ and $\mathrm{N}_{\mathrm{h}+\mathrm{l}_{\mathrm{2}}}$ are limited by $s / \sigma$ as $u$ becomes very large. In the growth case, $S / \sigma$ is an upper limit and in the decay case, it is a lower limit. By solving the equation

$$
\frac{1+P-u P}{1+P+u}=e^{-u}
$$

one finds the following formula for $P$.

$$
P=\frac{1-(1+u) e^{-u}}{u-1+e^{-u}},
$$

with

$$
P \approx 1-u / 3
$$

for small $u$ and

$$
P \approx 1 /(u-1)
$$

for large u. Also, P can be shown to be monotonely decreasing.

For several reasons, the exponential method is not a grod method to use. Constant $S$ in each interval is a rather crude approximation. Also, it is time consuming to compute $e^{-0}$ and then $P$ from Eq. (3.63), especially since this has to be done for every interval. Finally, the error in the method is controlled by $u$ rather than $u^{\prime}$ which is not good because often $u^{\prime} \ll \downarrow$ in much of the mesh. To some degree, the same disadvantages apply to $P$ based on high-order approximations to $\mathrm{e}^{-\mathrm{u}}$, Padé-type or other types. Note that in all these cases $P$ varies with $u$ so that Theorem 3.1 does not apply.
The limit version of the modifled diamond scheme is defined as follows. $N$ and $N_{h+\frac{1}{2}}$ are computed from Egs. (3.32) and (3.33) with $P=1$. In most cases these are final values for the interval. But if $\mathrm{N}_{\mathrm{h}+\frac{1}{2}}>(2-\beta) \mathrm{S} / \sigma$ occurs in the decay case, $\mathrm{N}_{\mathrm{h}+\mathrm{l}_{2}}$ is set by $\mathrm{L}_{\mathrm{h}+\mathrm{l}_{2}}$, the limit used in the test.

$$
\mathrm{N}_{\mathrm{h}+\mathrm{l}_{2}}=\mathrm{L}_{\mathrm{h}+\frac{1}{2}} \text {, }
$$

and $\mathrm{N}$ is computed from

$$
\mathrm{N}=\mathrm{S} / \sigma+\left(\mathrm{N}_{\mathrm{h}-\frac{1}{2}}-\mathrm{L}_{\mathrm{h}+\frac{3}{2}}\right) / \mathrm{u},
$$

whereupon $P$, If needed, can be computed from

$$
\begin{aligned}
P & =\left(N-N_{h+\frac{1}{2}}\right) /\left(N_{h-\frac{1}{2}}-N\right) \\
& =\frac{\left(N_{h-\frac{1}{2}}-L\right)+u(S / \sigma-L)}{(u-1)\left(N_{h-\frac{1}{2}}-L\right)-u(S / \sigma-L)} \\
& =\frac{N_{h-\frac{1}{2}}+u S / \sigma-(u+1) L}{(u-1) N_{h-\frac{1}{2}}-u S / \sigma+L} .
\end{aligned}
$$

The most restrictive limits are those based on Eqs. (3.60) and (3.61), that is, on setting $B=1$. It was shown earlier that $\beta$ can be relaxed to $\beta=\frac{1}{2}$ In the decay case. This is the motivation for introducing the coefficient $B$, and for using $2-\beta$ in the growth case. In the zero fix-up scheme, a 1 imit scheme used a great deal in the past, $B$ is set to zero. This scheme, however, is in clear violation of the physics, which probably explains why it sometimes failed to control adequately oscillatory tendencles in the numerical solutions.

A way to compare the MDS method with other methods is to examine how well exponential decay is approximated when $s / \sigma=0\left(u^{\prime}=u\right)$. A few approximations are listed in Table III-2.

In some situations it may be useful to accept negative $s / \sigma$ and, as a result, also negative $\mathrm{N}_{h-\frac{1}{2}}$. In most cases, this would occur for a few intervals, directions, and groups. This is not in any way upsetting in the MDS wethods. If both $\mathrm{N}_{h-\frac{1}{2}}$ and $\mathrm{s} / \sigma$ are negative in an interval, the procedure is not changed, the same formula for ' $u^{\prime}$ is used, etc. If one of these quantities is negative and the other positive, the only change is that $u$ ' may be larger than $u$, which is quite acceptable. If $\mathrm{N}$ from the 
TABLE III-2

APPROXIMATION TO $\mathrm{e}^{-\mathrm{u}}$ IN VARIOUS METHODS

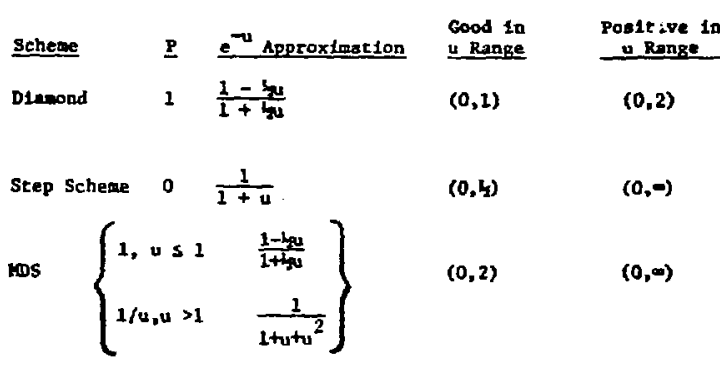

DS calculation is equal to zero, one sets $P=0$.

Growth and decay of flux are interpreted as increase or decrease in magnitude.

In conclusion, therefore, the modified diamond scheme (MDS) seems to take care of the difficulties of the past, those encountered with the straight diamond scheme (DS) or with improperly defined MDS schemes. It does not increase the requirements for computer storage and, although the computing time per cell is increased slightly, the total time is 11kely to be less because of the decreased interference with the inner iteration convergence resulting from the smoother MDS solutions.

\section{SOLUTION IN SPHERICAL GEOMETRY}

The solution of the transport equation in curviInear geometries, compared to solution in rectangulat cases, is more involved. Here, starting with spherical geometry, the partial differential equation is given by

$$
\begin{aligned}
& \mu \frac{\partial}{\partial r} A(r) N(r, \mu)+A^{\prime}(r) \frac{\partial}{\partial \mu} \frac{1}{2}\left(1-\mu^{2}\right) N(r, \mu) \\
& +\sigma(r) A(r) N(r, \mu)=A(r) S(r),
\end{aligned}
$$

where

$$
A(r)=4 \pi r^{2}
$$

and

$$
A^{\prime}(r)=\frac{\partial}{\partial r} A(r)=8 \pi r
$$

This represents the conservation form of the equation because the coefficients $\mu$ and $A^{\prime}$, respectively, of $\frac{\partial}{\partial r}$ and $\frac{\partial}{\partial \mu}$ are independent of the variables of differentiation. Transfonned to the regular form, with terms in $\frac{\partial}{\partial r} N$ and $\frac{\partial}{\partial \mu} N, E q .(4.1)$ becomes

$$
\begin{aligned}
& \mu \frac{\partial}{\partial r} N(r, \mu)+\frac{1}{r}\left(1-\mu^{2}\right) \frac{\partial}{\partial \mu} N(r, \mu) \\
& +\sigma(r) N(r, \mu)=S(r),
\end{aligned}
$$

where the $1 / \mathrm{r}$ coefficient comes from

$$
\frac{1}{2} A^{\prime}(r) / A(r)=1 / r
$$

In the discrete ordinates formulation, Eq. (4.1) is written

$$
\begin{aligned}
& \mu_{m} \frac{\partial}{\partial r} A(r) N_{m}(r) \\
& +\frac{A^{T}(r)}{w_{m}}\left[\alpha_{m+\frac{1}{2}} N_{m+\frac{1}{2}}(r)-\alpha_{m-\frac{1}{2}} N_{m-\frac{1}{2}}(r)\right] \\
& +\sigma(r) A(r) N_{m}(r)=A(r) S(r)
\end{aligned}
$$

where $w_{m}=\frac{1}{2} \Delta \mu_{m}$ and $\alpha_{m-\frac{1}{2}}, m=1,2, \ldots, n+1$, are coefficients to be determined.

$$
\alpha_{m-\frac{1}{2}} \approx \frac{1}{4}\left(1-\mu_{m-\frac{1}{2}}{ }^{2}\right),
$$

with equality specified for $m=1$, for which $\mu_{m-\frac{1}{2}}=$ - 1, and hence

$$
\alpha_{\frac{1}{2}}=\frac{1}{4}\left(1-\mu_{\frac{1}{2}}^{2}\right)=0
$$

The terms in $\alpha_{m+\frac{1}{2}}$ and $\alpha_{m-\frac{1}{2}}$ have the meaning of losses and gains, respectively, to the $\mathrm{m}^{\text {th }}$ discrete ray, or bundle of particles, tn the course of streaming. As a consequence of streaming, such changes can occur in a system described by curvilinear coordinates. In such streaming, the value of $\mu$ assoctaiod with the moving particle depends on $r$ and 1 s monotonely increasing.

The differential operator in Eq. (4.6), as in Eq. (4.1), must clearly vanish in any situation with constant $N$. This condition on the derivative ceims yfelds the following recursion for $\alpha$.

$$
\alpha_{m+\frac{1}{2}}-\alpha_{m-\frac{1}{2}}=-w_{m} \mu_{m}
$$

Therefore, $\alpha_{3 / 2}=-w_{1} \mu_{1}$, where $\mu_{1}$ is che discrete 
$\mu$ that is closest $L \mu=-1$, and $\alpha_{m+\frac{l}{2}} \geq 0$ for all $m$. Suming both sides of Eq. (4.9) over $m$, orie derives

$$
\alpha_{n+\frac{\alpha_{2}}{2}}-\alpha_{r_{2}}=-\Sigma_{m} w_{m} \mu_{m}=0
$$

where the second equality comes from Eq. (3.5) and the last $\alpha, \alpha_{n+\delta_{2}}$, equals zero. The midpoint $\alpha_{m}$ is defined by

$$
\alpha_{m}=\frac{1}{2}\left(\alpha_{m+r_{2}}+\alpha_{m-\frac{1}{2}}\right) .
$$

Note that if the quadrature is symmetric in the sense discussed in Sec. III, the coefficients are also symmetric, $\alpha_{n+\frac{1}{2}}=\alpha_{1 / 2}=0, \alpha_{n}=\alpha_{1}, \alpha_{n-\frac{1}{2}}=\alpha_{3 / 2}$, $\alpha_{n-1}=\alpha_{2}$, etc., with

$$
a_{\frac{1}{2}(n+1)}=\frac{3}{2} \sum_{\mathrm{n}} w_{\mathrm{m}} w_{\mathrm{m}} \mid=\frac{1}{4} \text {. }
$$

In solving Eq. (4.6) numerically, the progression is clearly from smaller $\mu_{m}$ toward larger $\mu_{m}$, and is, as in the infintte plane case for $x$, in the $r$ direction indicated by the sign of $\mu$, that is, inward for negative $\mu$ and outward for positive $\mu$. The first ray equation ( $m=1$ ) possesses no streaming source, since $\alpha_{\frac{3}{2}}=0$, and the last ray equation $(m=n)$ no streaming sink, since $a_{n+\frac{1}{2}}=0$. However, because $\mathrm{N}_{\mathrm{m}+\mathrm{l}_{\mathrm{z}}}$ is obtained by extrapolation from $\mathrm{N}_{\mathrm{m}}$ and $\mathrm{N}_{\mathrm{m}+\mathrm{k}_{2}}$, values for $\mathrm{N}_{\frac{1}{2}}$ for an Inftializing ray are needed. The equation for $\mathrm{N}_{\frac{1}{2}}$, obtained from Eq.

(4.4) by setting $\mu=-1$, is

$$
-\frac{\partial}{\partial r} N_{\frac{1}{2}}(r)+\sigma(r) N_{\frac{1}{2}}(r)=S(r)
$$

Next, by a process of adding and subtracting terms (shown in detail later), and by setting

$$
N_{m}(r)=\frac{1}{2}\left[N_{m-\frac{1}{2}}(r)+N_{m+\frac{1}{2}}(r)\right],
$$

Eq. (4.6) can be put in a regular form, as follows.

$$
\begin{aligned}
& u_{m} \frac{\partial}{\partial r} N_{m}(r)+\frac{2 \alpha_{m}}{r w_{m}}\left[N_{m+\frac{s}{2}}(r)-N_{m-\frac{1}{2}}(r)\right] \\
& +\sigma(r) N_{m}(r)=S(r) .
\end{aligned}
$$

The balance and net flux equations for continuous and discrete $\mu$, respectively, are obtained on the basis of the defintions

$$
\bar{N}(r)=\frac{1}{2} \int_{-1}^{1} N(\Sigma, \mu) d \mu=\Sigma_{m} w_{m} N_{m}(r)
$$

and

$$
I(r)=\frac{1}{2} \int_{-1}^{1} N(r, \mu) \mu d \mu=\Sigma_{m} w_{m} \mu_{m} N_{m}(r),
$$

and the quadrature operators

$$
\frac{1}{2} \int_{-1}^{1} 3 \mu()=\Sigma_{\text {m }} w_{m}()
$$

and

$$
\frac{3}{2} \int_{-1}^{1} \mu d \mu()=3 \Sigma_{m} w_{m} \mu_{m}() .
$$

The operators in Eq. (4.18) are applied to Eqs. (4.1) and (4.6), respectively, and the operators in Eq. (4.19) to Eqs. (4.4) and (4.15). In applying Eq. (4.19) to the continuous and discrete cases, respectively, the diffusion approximations

$$
N(\mathbf{r}, \mu)=\bar{N}(\mathbf{r})+3 \mu I(r)
$$

and

$$
N_{m}(r)=\bar{N}(r)+3 \mu_{m} I(r)
$$

are also used.

In applying Eq. (4.18) to Eq. (4.6), the terms in $\alpha$ vanish since

$$
A^{\prime}(r) \sum_{m}\left(\alpha_{m+\frac{1}{2}} N_{m-\frac{1}{2}}-\alpha_{m-\frac{1}{2}} N_{m-\frac{1}{2}}\right)=0
$$

because of cross cancellation and $\alpha_{n+\frac{1}{2}}=\alpha_{\frac{1}{2}}=0$. Also, after applying Eq. (4.19) to Eq. (4.15), the terms in $\alpha$ in that equation vanish since

$$
(6 / r) \sum_{m} \mu_{m} \alpha_{m}\left(N_{m+\frac{1}{2}}-N_{m-\frac{1}{2}}\right)=0,
$$

first because, by using Eq. (4.21), the aifierence of the N's is replaced by $6 \omega_{m} I$ and second because $\alpha_{m}$, 11ke $w_{m}$ is an even function in $\mu$. As a result of the above, the balance and net flux equations are given by

$$
\frac{\partial}{\partial r} A(r) I(r)+\sigma(r) A(r) \bar{N}(r)=A(r) S(r)
$$




$$
\frac{\partial}{\partial r} \bar{N}(r)+3 \sigma(r) I(r)=0
$$

for the discrete ordinates as well as the analytical case. Note that the construction or unsymmetrlc quadrature sets is not simple since $\mu_{\mathrm{m}}$ selections must satisfy the condition

$$
\sum_{m} w_{m} \mu_{m} \alpha_{m}=0
$$

with $\alpha_{\text {m }}$ depending on $\mu_{s}, s \leq m$, through Eqs. (4.9) and (4.11).

The finite cell formulation of the transport equation for sphere, derived independently of Eq. (4.6), is given by

$$
\mu_{m}\left(A_{1+\frac{1}{2}} N_{m, 1+\frac{1}{2}}-A_{1-\frac{1}{2}} N_{m, 1-\frac{1}{2}}\right)+\left(C_{1} / w_{m}\right)\left(\alpha_{m+\frac{1}{2}} N_{m+\frac{1}{2}, i}-a_{m-\frac{1}{2}} N_{m-\frac{1}{2}, i}\right)+\sigma_{1} v_{1} N_{m, 1}=v_{1} S_{1},
$$

where

$$
\begin{aligned}
& A_{i+\frac{1}{2}}=4 \pi r_{i+\frac{1}{2}}{ }^{2}, \\
& A_{i}=\frac{1}{2}\left(A_{1+\frac{1}{2}}+A_{1-\frac{1}{2}}\right)=4 \pi\left(\bar{r}_{1}^{2}+\frac{1}{4} \Delta r_{i}{ }^{2}\right), \\
& C_{1}=A_{i+\frac{1}{2}}-A_{1-\frac{1}{2}}=8 \pi \bar{r}_{i} \Delta r_{i},
\end{aligned}
$$

and

$$
\begin{aligned}
v_{1} & =(4 \pi / 3)\left(r_{1+d_{2}}^{3}-r_{1-d_{2}}^{3}\right) \\
& =4 \pi\left(\bar{r}_{1}^{2}+\frac{1}{12} \Delta r_{1}^{2}\right) \Delta r_{1},
\end{aligned}
$$

with

$$
\Delta r_{1}=r_{1+\frac{1}{2}}-r_{1-\frac{1}{2}}
$$

and

$$
\bar{r}_{t}=\frac{1}{2}\left(r_{i-k_{2}}+r_{i+1 / 2}\right)
$$

Here the relations

$$
a_{m+\frac{1}{2}} / w_{m}=\alpha_{m} / w_{m}-\frac{1}{2} \mu_{m}
$$

and

$$
\alpha_{m-\frac{1}{2}} / w_{m}=\alpha_{m} / w_{m}+\frac{1}{2} \mu_{m}
$$

were used. For further simplificacion, the following formulas are useful.

$$
A_{i+\frac{1}{2}}-V / \Delta r=\frac{1}{2} C+(A-V / \Delta r)
$$

and

$$
v / \Delta r-A_{1-\frac{1}{2}}=\frac{1}{2} C-(A-V / \Delta r)
$$

To obtain the desired consistency between Eqs. (4.15) and (4.35), the terms in the second Iine of Eq. (4.35) plus the term in $-\frac{1}{2} \mu$, wust vanish. This leads to the following difference relation.

In simplified notation, Eq. $(4.27)$ is written

$$
\mu\left(A_{1+\frac{1}{2}} N_{1+\frac{1}{2}}-A_{1-\frac{1}{2}} N_{i-\frac{1}{2}}\right)+(C / w)\left(\alpha_{m+\frac{1}{2}} N_{m+\frac{1}{2}}-\alpha_{m-\frac{1}{2}} N_{m-\frac{1}{2}}\right)+\sigma V N=v s .
$$

In the finite cell iquation, $s_{i}, N_{m, 1}, \sigma_{1} N_{m, 1}$, and $C_{1} N_{m, i}$ represent volume average of $S(r), N_{m}(r)$, 


$$
\begin{aligned}
& \frac{1}{2}\left(N_{m+\frac{1}{2}}+N_{m-\frac{1}{2}}\right)=\left(\frac{1}{2}+\Delta r / 12 \bar{r}\right) N_{1+\frac{1}{2}} \\
& +\left(\frac{1}{2}-\Delta r / 12 \bar{r}\right) N_{1-\frac{1}{2}}
\end{aligned}
$$

where the coefficlent $\Delta r / \bar{r} \bar{r}$ comes from

$$
(\mathrm{A}-\mathrm{V} / \Delta \mathbf{r}) / \mathrm{C}=\Delta \mathbf{r} / 12 \overline{\mathbf{r}} \text {, }
$$

which has its maximum value of $1 / 6$ for the first $r$ interval $(0, \Delta r)$. With this difference relation, Eq. (4.35) simplifies to

$$
\begin{aligned}
& \mu\left(N_{1+\frac{1}{2}}-N_{1-\frac{1}{2}}\right)+(C \alpha \Delta r / V w)\left(N_{m+1}-N_{m-\frac{1}{2}}\right) \\
& +\sigma \Delta r N=\Delta r S
\end{aligned}
$$

as the finite cell equivalent to Eqs. (4.4) and $(4.15)$. If one multiplies the terms by $w$ and takes Arw as the volume element, Eq. (4.42) may be regarded as the rectangularized version of Eq. (4.27). For the special straight-in direction, $\mu=-1$, with $\alpha=0$ erasing the $\alpha$ term in the equation, the result is a planar-type equation.

$$
-\left(N_{1+\frac{1}{2}}-N_{1-\frac{1}{2}}\right)+\sigma \Delta r N=\Delta r S,
$$

corresponding to Eq. (4.13). The same equation is obtained from Eq. (4.34) by setting $\mathrm{N}_{m+t_{2}}=\mathrm{N}_{\mathrm{m}-\frac{1}{2}}$, $\alpha_{m+\frac{1}{2}} / w=1$ (the correct $11 n i t$ for a small $\mu$ interval near $\mu=-1)$, and $\alpha_{m-\frac{1}{2}}=0$, and then eliminacing $\mathrm{N}_{\mathrm{m}+\frac{1}{2}}$ by means of Eq. $(4,40)$.

The above analysis shows that there are special restraints on the difference relations in curved geometries. In the spherical case, one can select one difference relation in the usual manner, foining this with Eq. $(4.40)$, the principal relation. Subsequently, upon substitution of these relations, Eq. $(4.42)$ can be solved for tts three unknowns, $N_{1}, N_{m+\frac{1}{s}}$, and $N_{h+\frac{1}{2}}$, where $N_{h+\frac{1}{2}}=N_{1+\frac{1}{2}}$ for $\mu>0$, and $N_{h+\frac{1}{3}}-N_{1-\frac{1}{2}}$ for $\mu<0$. Truncation error analysis (as made in Refs. 2 and 3 ) suggest tie following scheme as a supplement to Eq. $(4,40)$.

$$
N=\left(\frac{1}{2}+\Delta r / 12 \bar{r}\right) N_{1+\frac{1}{2}}+\left(\frac{1}{2}-\Delta r / 12 \bar{r}\right) N_{1-y_{2}} \cdot(4.44)
$$

a weighted scheme, which in combination with Eq. (4.40) gives

$$
\begin{aligned}
N & =\frac{1}{2}\left(N_{m+\frac{1}{2}}+N_{m-b_{2}}\right) \\
& =\left(\frac{1}{2}+\Delta r / 12 \bar{r}\right) N_{1+\frac{1}{2}}+\left(\frac{1}{2}-\Delta r / 12 \bar{r}\right) N_{1-\frac{1}{2}}
\end{aligned}
$$

where, moreover, the first equality is consistent with Eq. (4.14). This total scheme may be completed for $P<1$ by replacing $N$ in the above by

$$
\frac{1}{2}(1+P) N+\frac{1}{2}(1-P) N_{m-\frac{1}{2}} .
$$

The scheme defined by Eq. $(4.44)$, involving unequal weights, does not seem proper, however, for the siraight-in case, Eq. (4.43). It seems that the total scheme should be replaced, in some way, by

$$
N=\frac{1}{2}\left(N_{1+\frac{1}{2}}+N_{1-\frac{1}{2}}\right)+\frac{1}{2}\left(N_{m+\frac{1}{2}}+N_{m-\frac{1}{2}}\right) .
$$

By a slightly different procedure, partly empirical but in other respects like that used to esrablish Eq. $(4.40)$, one can indeed (as shown in Ref. 1) cerminate by Eq. $(4.47)$. This involves the replacements

$$
\sigma \mathbf{A} \mathbf{r} / \mathbf{V}+\sigma
$$

and

$$
S / \sigma+(A \Delta r / V-1) \bar{N}+S / \sigma
$$

This method, which manipulates the source and sink terms so that the number of particles is preserved, is not perfect as applied to Eq. $(4.43)$, but it is probably better than the method based on Eq. (4.45). Some of the earlier methods for coping with the diffusion condition should be mentioned. These methods, which were unsatisfactory for other reasons, involved the manipulation of area and volume elements, cumputsng, for example, $A_{i+b_{1}}$ from the exact $v_{i}$ using the recursion

$$
A_{1+\frac{1}{2}}=2 v / \Delta r-A_{1-\frac{1}{2}}
$$

so that $A \Delta r=V$, or computing $V$ from the exact $A^{\prime}$ 's by

$$
V=\Delta \Delta r
$$

The method of claracteristics resolves the above problem very nicely, at least analyctcally. Final Judgment on this approach must be reserved until a 
number of numerical tests have been made. In characteristic from, $\mathrm{Eq} .(4.34)$ is written

$$
\left(N_{h+\frac{1}{2}}-N_{h-\frac{1}{2}}\right)+u N=u s / \sigma,
$$

where

$$
u=\sigma / T .
$$

Here $T$ has the meaning of total effective ourflow area (eqral to inflow area) of the cell, being the sum of the individual areas (in this case $|\mu| A_{1+\frac{1}{2}}$ and $\left.\mathrm{C} \alpha_{m+1 / 2} / w\right)$ and is given by

$$
\begin{aligned}
T & =|\mu| A_{1+\frac{1}{2}}+C a_{m+\frac{1}{2}} / w \\
& =|\mu| A_{1-\frac{1}{2}}+C \alpha_{m-\frac{1}{2}} / w \\
& =|\mu| A+C \alpha / w .
\end{aligned}
$$

These expressions, and those below, are for positive $\mu$; for negative $\mu, 1+\frac{1}{2}$ is replaced by $1-\frac{1}{2}$ and $1-\frac{1}{2}$ by $1+t_{2}$. Also, if Eq. (4.52) is to be equivalent to Eq. (4.34), $N_{h+\frac{1}{2}}$ and $N_{h-\frac{1}{2}}$, the average outflow and Inflow for the cell must be defined by

$$
\mathrm{TN}_{h+\frac{1}{2}}=|\mathrm{H}| \mathrm{A}_{1+\frac{1}{2}} \mathrm{~N}_{1+\frac{1}{2}}+\left(\mathrm{Ca} \mathrm{m}_{\mathrm{m}+\frac{1}{2}} / \mathrm{w}\right) \mathrm{N}_{\mathrm{m}+\frac{1}{2}}
$$

and

$$
\mathrm{TN}_{h-\frac{1}{2}}=|\mu| A_{1-\frac{1}{2}} \mathrm{~N}_{i-\frac{1}{2}}+\left(\mathrm{C} \alpha_{m-\frac{1}{2}} / w\right) \mathrm{N}_{n-\frac{1}{2}} \text {, }
$$

respectively. To solve Eq. (4.52), one introduces the difference relation

$$
N_{h+d_{2}}=(1+P) N-P N_{h-d_{2}}
$$

and proceeds as in Sec. III to obtain $P, N$, and $\mathrm{N}_{\mathrm{h}+\frac{1}{2}}$. Then to detail the output, given $\mathrm{N}_{\mathrm{h}+\frac{1}{2}}$, one uses the definition of $N_{h+\frac{1}{2}}$ in terms of $N_{1+\frac{1}{2}}$ and $N_{m+\frac{1}{2}}$ or $N_{1-1 / 2}$ and $N_{m+l_{2}}$, as the case may be, plus the difference relation Eq. (4.40). The atraight-in case is handled somewhat differently because Eq. (4.40) implies Eq. $(4.43)$. Therefore, in tlids case

$u=\sigma \Delta \mathbf{r} / \mathrm{T}$,

with $T=1, N_{h+\frac{1}{3}}=N_{1-\frac{1}{2}}$ and $N_{h-\frac{1}{2}}=N_{1+\frac{1}{3}}$. Detalling of outputs is done as follows. For the straight-in direction it remains to calculate $N_{1,1}$, the Initial function for extrapolation in $\mu$.

$$
\mathrm{N}_{\frac{\mathrm{l}}{2}}=\left(\frac{1}{2}+\Delta \mathrm{r} / 6 \overline{\mathrm{r}}\right) \mathrm{N}_{\mathrm{h}-\frac{1}{2}}+\left(\frac{1}{2}-\Delta \mathrm{r} / 6 \overline{\mathrm{r}}\right) \mathrm{N}_{\mathrm{h}+\frac{1}{2}} \cdot
$$

For the case of negative $\mu$,

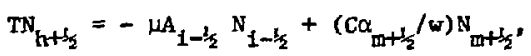

combined with Eq. $(4.40)$ to eliminate $\mathrm{N}_{m+\frac{1}{2}}$, gives

$$
N_{i-\frac{1}{2}}=\frac{N_{h+\frac{1}{2}}+\left(C \alpha_{m+\frac{1}{2}} / T w\right)\left[N_{m-\frac{1}{2}}-(1+\Delta r / 6 \bar{r}) N_{i+\frac{1}{2}}\right]}{1-\left(C \alpha_{m+\frac{1}{2}} / T w\right)(\Delta r / 6 \bar{r})} .
$$

Since for $1=1$, the factors in the denominator equal 1 and $1 / 3$, respectively, the equation simplifies in this case to

$$
N_{m, \frac{1}{2}}=\frac{3}{2}\left(N_{h+\frac{1}{2}}+N_{m-\frac{1}{2}}\right)-2 N_{1+\frac{1}{2}} \text {. }
$$

For the positive $\mu$ directions,

$$
T N_{h+\frac{1}{2}}=\mu A_{1+\frac{1}{2}} N_{1+d_{2}}+\left(C \alpha_{m+\frac{1}{2}} / w\right) N_{m+\frac{1}{2}}
$$

combined with Eq. $(4.40)$ to eliminate $\mathrm{N}_{\mathrm{m}+\frac{1}{2}}$, gives

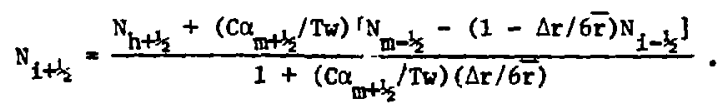

For $m=n$, in which case $\alpha_{m+\frac{1}{2}}=0$, this reduces to $N_{i+y_{3}}=N_{h+\frac{1}{2}}$. After the extrapolation in the r-direction has been done, $N_{m+i}$ is calculated from

$$
N_{m+d_{2}}=(1+\Delta r / 6 \bar{r}) N_{1+\frac{1}{2}}+(1-\Delta r / 6 \bar{r}) N_{1-l_{2}}-N_{m-\frac{1}{2}}
$$

The final step in the calculation is the correction for skewing as described in Sec. II.

v. SOLUTION IN CYLINDRICAL GEOMETRY

In Infinite cylindrical (r) geometry with two variables for direction, the radial and axtal components $\mu$ and $\xi$, the transport equation 18 given by

$$
\begin{aligned}
& \mu \frac{\partial}{\partial r} A(r) N(r, \mu, \xi)-A^{\prime}(r) \frac{\partial}{\partial \phi} \eta N(r, \mu, \xi) \\
& +\sigma(r) A(r) N(r, \mu, \xi)=A(r) S(r)
\end{aligned}
$$

where $A$ is the area element 


$$
A(\mathbf{r})=2 \pi r,
$$

with $A^{\prime}(r)=2 \pi$, and where $\phi$ and $\eta$ are related to $\mu$ and $\xi$ by

$$
\mu=\sqrt{1-\xi^{2}} \cos \phi
$$

and

$$
n=\sqrt{1-\xi^{2}} \sin \phi
$$

with $\pi \geq \phi \geq 0$ and $0 \leq \xi \leq 1$. It follows from this that $-1 \leq \mu \leq 1$ and $0 \leq n \leq 1$, and that the domain of $\Omega$ is comprised of two octants of the unit sphere. Note that if $N(r, \mu, \xi)$ is constant, the differential operator in Eq. (5.1) vanishes because

$$
\mu \frac{\partial}{\partial r} A(r)-A^{\prime}(r) \frac{\partial}{\partial \phi} \eta=0 .
$$

As in the spherical case, the discretization of Eq. (5.1) is accomplished by means of quadrature methods and difference schemes. Numerical quadrature combines with finite intervals in $\mu$ and $\xi$, and difference methods combine with finte intervals in $r$. The construction is such that in the limit of small intervals the discrete and analytical equations are consistent. An intermediate step in the construction is the discrete ordinates formulation where the treatment is discrete in $\mu$ and $\xi$ and cortinuous in $r$.

A number of equations are involved in the consistency arguments: first the conservation equation [Eq. (5.1) and its alternate, regular form, Eq. (5.6) below, second the basic derived equations, the balance and net flux equations [Eqs. (5.7) and $(5.8)$ belowl, and third the associated diffusion approximation [Eq. (5.9)] used in the derivation of Eq. (5.8). These equations are given by

$$
\begin{aligned}
& \mu \frac{\partial}{\partial r} N(r, \mu, \xi)-(\eta / r) \frac{\partial}{\partial \phi} N(r, \mu, \xi) \\
& +\sigma(r) N(r, \mu, \xi)=S(r), \\
& \frac{\partial}{\partial r} A(r) I(r)+\sigma(r) A(r) \bar{N}(r)=A(r) S(r), \\
& \text { (1/3) } \frac{\partial}{\partial r} \bar{N}(r)+\sigma(r) I(r)=0 .
\end{aligned}
$$

$$
\mathrm{N}(r, \mu, \xi) \approx \overrightarrow{\mathrm{N}}(\mathbf{r})+3 \mu \mathrm{I}(\mathrm{r}) .
$$

In the above, the scalar flux $\bar{N}(r)$ and current $I(r)$, two functionals of $N(r, \mu, \xi)$ may be defined as the result of two operators.

$$
F_{0}=\frac{1}{\pi} \int_{0}^{1} d \xi \int_{0}^{\pi} d \phi
$$

and

$$
F_{1}=\frac{1}{\pi} \int_{0}^{1} d \xi \int_{0}^{\pi} \mu d \phi
$$

applied to $N(r, \mu, \xi)$.

Equation (5.6) is found by continued differentiation in Eq. (5.1); Eq. (5.7) by applying the first operator [Eq. $(5.10)]$, term by term to Eq. (5.1); and Eq. (5.8) by applying the second operator after substitution of Eq. (5.9), However, Eq. (5.8) is mnre readily obtained if the second operator [Eq. (5.11)] is applied to Eq. (5.6) rather than to $\mathrm{Eq} .(5.1)$.

In construcing the discrete ordinates formulation, one starts w!.th

$$
\begin{aligned}
& \mu_{\ell, m} \frac{\partial}{\partial r} A(r) N_{\ell, m}(r) \\
& +\frac{A^{\prime}(r)}{w_{\ell, m}}\left[\alpha_{\ell, m+\frac{1}{2}} N_{\ell, m+\frac{d_{2}}{2}}(r)-\alpha_{\ell, m-\frac{1}{2}} N_{\ell, m-\frac{1}{2}}(r)\right] \\
& +\sigma(r) A(r) N_{\ell, m}(r)=A(r) S(r)
\end{aligned}
$$

where $\ell$ is the level index (the index associated with $\xi$ ) and $m$ the $\mu$ (or $\phi$ ) Index, where the quant 1ties $\mu_{\ell, \text { m }}$ are discrete values of $\mu$ depending on $\xi$ as well as $\phi$, and where the second term approximates the term in Eq. (5.1) with the $\$$ derivative. The meaning of $\mathrm{N}_{,}, \mathrm{m}$ ( $r$ ) is

$$
N_{\ell, m}(r)=N\left(r, \mu_{\ell, m}, \xi_{\ell, m}\right)
$$

and weights $w_{\ell, m}$, together with the points or nodes, $\left(\mu_{\ell, m}, \eta_{\ell, m}, \xi_{\ell, m}\right)$ constitute the quadrature set.

By requiring that the differential operator in Eq. $(5,12)$ vanish in the case of constant $N$, as for the analytical equation, the following recursion formula for $\alpha$ is obtained.

$$
\alpha_{\ell, m+\frac{1}{2}}-\alpha_{\ell, m-\frac{1}{2}}=-w_{\ell, m} \mu_{\ell, m}
$$


which can be used to compute $\alpha$ because $\alpha_{\ell, \frac{1}{2}}$ can be set to zero as seen later.

In the following, the point weights $w_{\ell, m}$ are assumed to be equal.

$$
w_{\ell, m} \equiv w=4 / n(n+2)
$$

for ary given order $n$ of approximation, $n=2,4$, $\ldots$. The associated nodes $\left(\mu_{\ell, m}, \eta_{\ell, m}, \xi_{\ell, m}\right)$ are assumed to be arranged in a triangular fashion on $\frac{1}{2} \mathfrak{n}$ levels with $n=2 \ell+2$ nodes on level $\ell, m=1$, $2, \ldots, n-2 \ell+2, \ell=1,2, \ldots, 1-2 n$. This yields a total of $n(n+2) / 4$ nodes, with $n(n+2) / 8$ per octant. From this, with the level weights $W_{\ell}$ defined by

$$
w_{\ell}=\Sigma_{m} w_{\ell, m}=(n-2 \ell+2) w,
$$

the normalization is

$$
\Sigma_{\ell, \mathrm{m}} \mathbf{w}_{\ell, \mathrm{m}}=\Sigma_{\ell} w_{\ell}=1
$$

It is further assumed that the nodes are chosen so that

$$
\mu_{\ell, \mathrm{m}}^{2}+r_{\ell, \mathrm{m}}^{2}+\xi_{\ell, \mathrm{m}}^{2}=1
$$

and

$$
\Sigma_{\ell, \mathrm{m}} w_{\ell, \mathrm{m}} w_{\ell, \mathrm{m}}=\Sigma_{\ell, \mathrm{m}} w_{\ell, \mathrm{m}} \xi_{\mathrm{m}}^{2}=1 / 3,
$$

$\mathrm{m}=1,2, \ldots, \mathrm{n}-2 \ell+2, \ell=1,2, \ldots, \mathrm{b} n$, from which it follows that

$$
\Sigma_{\ell, \mathrm{m}} w_{\ell, \mathrm{m}} n_{\ell, \mathrm{m}}^{2}=1 / 3
$$

Defining the level cosines $\bar{\xi}_{\ell}$ by

$$
\bar{\xi}_{\ell}=\sqrt{\left(z_{m} w_{\ell, m} \xi_{\ell, m}\right) / w_{\ell}},
$$

it also follows that

$$
\Sigma_{\ell} w_{\ell} \bar{\xi}_{\ell}^{2}=1 / 3
$$

Finally, it is assumed that if $\left(\mu_{\ell, m} \eta_{\ell, m} \xi_{\ell, m}\right)$ ie a node in the set, so is $\left(-\mu_{\ell, m}, \eta_{\ell, m}, \xi_{k_{,}, \mathrm{m}}\right)$. With this sign reversal symmetry and equal weights, it follows that

$$
\Sigma_{\ell, m} w_{l, m} \mu_{\ell, m}=0
$$

and also that $\alpha_{\ell, m}$ is an even function of $\nu_{\ell, m}$ for each $\ell$. As a consequence,

$$
\Sigma_{\ell, \mathrm{m}} \omega_{\ell, \mathrm{m}} \mu_{\ell, \mathrm{m}} \alpha_{\hat{\ell, m}}=0 .
$$

As in the spherical case, the basic quadrature set is augmented by special zero-weight points, one for each $\xi$ level, say for $m=1$. This changes the m sequence to $r .=1,2, \ldots, n=2 \ell+3$ and the totality of points to $n(n+4) / 4$. For the special points one has $w_{\ell, 1}=0, n_{\ell, 1}<0, \xi_{\ell, 1}=\sqrt{1-\mu_{\ell, 1}{ }^{2}}$, and $\alpha_{3 / 2}=\alpha_{1}=\alpha_{1 / 2}=0$. The indices for the nodes with negative $\mu$ are $m=2,3, \ldots, \frac{1}{2 n}-\imath+2$.

Returning to the discrete ordinates formulation, one observes that $w_{\ell, \text { m }}$ can be related to $\Delta \phi$ by

$$
w_{\ell, m}=-\left(w_{\ell} / \pi\right) \Delta \phi .
$$

Therefore, if Eq. (5.12) is to be consistent with Eq. (5.1), the $\alpha$ coefficients as computed from Eq. (5.14) must satisfy

$$
x_{\ell, \pi-\frac{1}{2}} \approx\left(w_{\ell} / \pi\right) n_{\ell, m-\frac{1}{2}}
$$

as well as

$$
\alpha_{\ell, m}=\frac{1}{2}\left(\alpha_{\ell m n+\frac{1}{2}}+\alpha_{\ell, m-\frac{l}{2}}\right) \geq\left(w_{\ell} / \pi\right) n_{\ell, \ldots},
$$

and do this with increasing accuracy as $n$ increases. in the one-dimensional case for spheres, the corresponding relations are

$$
x_{m-\frac{1}{2}}=\frac{1}{4}\left(1-\mu_{m-\frac{1}{2}}{ }^{2}\right)
$$

and

$$
a_{m}=\frac{1}{4}\left(1-\mu_{m}^{2}\right) \text {. }
$$

Note that for the spectal points in which case $\eta_{\ell, \frac{1}{2}}=0$. Eq. $(5,26)$ justifies that $\alpha_{\ell, \frac{1}{2}}$ be set to zero.

The accuracy of the relations of Eqs. (5.26) and (5.28) are tested in the next section in the case of ES quadrature, an easily constructed quadrature dofined and tabulated in that section.

To put Eq. (5.12) In the regular form, the first term is differentiated with the resuit

$$
A(r) \mu_{R, m} \frac{\partial}{\partial r} N_{Q, m}(r)+\mu_{R, m} A^{\prime}(r) N_{R, m}(r)(5.30)
$$


where the first term is the desired term. Second, the terms in $\alpha$ can be written

$$
\frac{A^{\prime}(r)}{w_{\ell, m}} \alpha_{\ell, m}\left[N_{\ell, m+\frac{1}{2}}(r)-N_{\ell, m-\frac{1}{2}}(r)\right]+\left(\alpha_{\ell, m+\frac{l}{2}}-\alpha_{\ell, m-\frac{1}{2}}\right) \frac{A^{\prime}(r)}{2 w_{\ell, m}}\left[N_{\ell, m+\frac{l}{2}}(r)+N_{\ell, m-\frac{1}{2}}(r)\right] .
$$

Here the first term with the factor $\alpha_{\ell, \mathrm{m}}$ is the de-

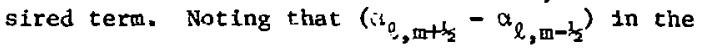
second term can be replaced by $-w_{\ell, m} ~^{\ell} \ell, m$, that term can be made to cancel the second term in Eq. (5.30) by assuming the difference relation

$$
N_{\ell, m}(r)=\frac{1}{2}\left[N_{\ell, m+\frac{1}{2}}(r)+N_{\ell, \mathbb{m}-s_{1}}(r)\right] .
$$

$$
\begin{aligned}
& A_{1}=\frac{1}{2}\left(A_{1+\frac{1}{2}}+A_{i-\frac{1}{2}}\right)=2 \pi \bar{r}_{i}, \\
& C_{i}=A_{i+\frac{1}{2}}-A_{1-\frac{1}{2}}=2 \pi \Delta r_{i},
\end{aligned}
$$

and

$$
v_{i}=\pi\left(r_{i+\frac{1}{2}}^{2}-r_{i-\frac{1}{2}}^{2}\right)=2 \pi \vec{r}_{i} \Delta r_{i}=A_{i} \Delta r_{i} .
$$

In this way, Eq. (5.12) is put in the regular form

$$
\mu_{\ell, m} \frac{\partial}{\partial r} N_{\ell, m}(r)+\frac{\alpha_{\ell, m}}{r w_{\ell, m}}\left[N_{\ell, m+\frac{1}{2}}(r)-N_{\ell, m-\frac{1}{2}}(r)\right]+\sigma(r) N_{0, m}(r)=s(r),
$$

consistent with Eq. (5.6).

Next, defining the discrete operators corresponding to Eqs. $(5.10)$ and $(5.11)$ by

$$
F_{0}=\Sigma_{\ell, m} w_{2, m}
$$

and

$$
F_{1}=\Sigma_{\ell, m}{ }^{w, m}, \mu_{\ell, m}
$$

$\vec{N}(r)$ and $I(r)$ may be defined as the result of these operators operating on $\mathrm{N}_{\mathrm{l}, \mathrm{m}}(\mathrm{r})$. Furthermore, on the basis of the properties assigned to the quadrature set, the operators Eqs. (5.34) and (5.35) applied to Eqs. (5.12) and (5.6), respectively, yield exactly Eqs. (5.7) and (5.8). In arriving at Eq. (5.8), Eq. (5.24) and the following discrete version of Eq. (5.9) are used.

$$
N_{\ell, \mathrm{m}}(r) \approx \bar{N}(r)+3 \mu_{\ell, m} I(r) .
$$

For the final step in the discretization, Eq. (5.12) is first writcen as a finite cell conservation law.
The correct balance equation,

$$
A_{1+d_{2}} I_{i+l_{2}}-A_{1-\frac{1}{2}} I_{i-\frac{1}{2}}+\sigma_{1} v_{1} \bar{N}_{1}=v_{1} s_{1},
$$

is obtained by applying the operators Eqs. (5.34) and (5.35) to Eq. (5.37). In this case $\bar{N}_{i}$ and $I_{i}$ are defined by

$$
\mathrm{N}_{1}=\Sigma_{\ell, \mathrm{m}} \mathrm{w}_{\ell, \mathrm{m}} \mathrm{N}_{\ell, \mathrm{m}, 1}
$$

and

$$
I_{1}=\Sigma_{\ell, \mathbb{m}} w_{\ell, m} \mu_{\ell, \mathbb{m}} N_{\ell, m 1} .
$$

respectively. To obtain the correct net flux equation,

$$
(1 / 3)\left(\overline{\mathrm{N}}_{1+\frac{1}{2}}-\overline{\mathrm{N}}_{1-\frac{1}{2}}\right)+\sigma_{i} \Delta \mathrm{r}_{i} \mathrm{I}_{i}=0,
$$

one uses Eq. (5.37) put in a regular form together with the diffusion approximation

$$
\begin{aligned}
\mu_{\ell, m} A_{1+\frac{1}{2}} N_{\ell, m, 1+\frac{1}{2}}-\mu_{\ell, m} A_{i-\frac{1}{2}} N_{\ell, m, 1-\frac{1}{2}} & +\left(C_{i} / w_{\ell, m}\right)\left(\alpha_{\ell, m+\frac{1}{2}} N_{\ell, m+\frac{1}{2}, 1}-\alpha_{\ell, m-\frac{1}{2}} N_{\ell, m-\frac{1}{2}, 1}\right) \\
& +\sigma_{1} v_{1} N_{\ell, m, 1}=v_{1} s_{1},
\end{aligned}
$$

where

$$
A_{1+\frac{1}{2}}=2 \pi r_{1+\frac{1}{2}},
$$




$$
\mathrm{N}_{\ell, \mathrm{m}, 1}=\overline{\mathrm{N}}_{1}+3 \mu_{\ell, \mathrm{m}} \mathrm{I}_{1}
$$

The regular form of Eq. (5.37) is found by adding and subtracting equal terms in that equation, obtaining

$$
\mu_{\ell, 1}=-\sum_{n=1}^{3_{2 n} n+1} w_{m}
$$

and in a chird method only one spectal direction is

$$
\begin{aligned}
& \mu_{\ell, m} A_{1}\left(N_{\ell, m, 1+\frac{l}{2}}-N_{\ell, m, 1-\frac{l}{2}}\right)+\frac{1}{2} \mu_{\ell, m}\left(A_{1+\frac{l}{2}}-A_{I-\frac{1}{2}}\right)\left(N_{\ell, m, i+\frac{1}{2}}+N_{\ell, m, i-\frac{l}{2}}\right) \\
& +\left(C_{1} \alpha_{\ell, m} / w_{\ell, m}\right)\left(N_{\ell, m+\frac{1}{2}, 1}-N_{\ell, m-\frac{l}{2}, 1}\right)-\frac{1}{2} \mu_{\ell, m} C_{i}\left(N_{\ell, m+\frac{1}{2,1}}+N_{\ell, m-\frac{1}{2}, i}\right)+\sigma_{1} v_{1} N_{\ell, m, 1}=v_{i} s_{1} .
\end{aligned}
$$

Here the first and second terms equal the first two terms in Eq. (5.37) and the two terms in $C_{i}$ equal the term in $C_{1}$ in Eq. (5.37). If the following difference is introduced,

$$
\begin{aligned}
& \frac{1}{2}\left(N_{\ell, m+\frac{1}{2}, 1}+N_{\ell, m-\frac{1}{2}, 1}\right) \\
& =\frac{1}{2}\left(N_{\ell, m, i+l_{2}}+N_{\ell, m, i-\frac{1}{2}}\right),
\end{aligned}
$$

the two terms in $\frac{1}{2} \mu_{\ell, m}$ in Eq. (5.47) cancel because $C_{1}=A_{1+\frac{1}{2}}-A_{1-\frac{1}{2}}$, and the proper regular difference equation, consistent with Eqs. (5.6) and $(5.33)$, is obtained.

$$
\mu_{\ell, m}\left(N_{\ell, m, 1+\frac{l}{2}}-N_{\ell, m, i-\frac{l}{2}}\right)+\frac{\Delta r_{1} \alpha_{\ell, m}}{\bar{r}_{1} w_{\ell, m}}\left(N_{\ell, i t+\frac{1}{2}, 1}-N_{\ell, m-l, 1}\right)+\sigma_{1} \Delta r_{1} N_{\ell, m, 1}=\Delta r_{1} s_{1},
$$

used, $\mu_{1,1}=-1.0$. In this sase, for $\ell>1$, one sets

$$
N_{\ell, 1}(r)=N_{\ell-1,5 / 2}(r)
$$

VI. ES $\mathrm{n}$ MECHANICAL QUADRATURE

The requirements or: numerical quadratire as used in transport calculations have been discussed in previous sections as well as in several earlier reports. ${ }^{4,5}$ In this section a simple quadrature method labeled ES $n$ is duscribed based on equal point weights and on approximating rather then precisely after dividing through by $A_{1}$ noting that $C_{1} / A_{1}=$ $\Delta r_{1} / r_{i}$ and $v_{1} / A_{1}=\Delta r_{1}$. Equation (5.49) corresponds to $\mathrm{Eq} .(4.40)$ in the spherical case. Note that the relation is simpler in cylindrical geometry because $v_{i}=A_{1} \Delta r_{i}$. The remaining steps in finding solutions in the cylindrical case by the method of characteristics parallel and are almost ident1cal to the steps in spherical geometry. These are described in Sec. IV beginning with the paragraph of Eq. (4.52). The difference in the equations is that the terms in $\Delta r / 6 \bar{r}$ in the spherical case are not present in the cylindrical case.

For the choice of special directions, more than one method is available. In a first method, $\mu_{\ell, I}$ is computed from

$$
\mu_{\ell, 1}=-\sqrt{1-\bar{\xi}_{\ell}^{2}}
$$

in a second, from satisfying a set of moment conditions. The simplicity comes from the fact that no solution of a system of nonlinear (or Iinear) equations is involved. Here $n$ controls the accuracy of the quadrature, $n=$ $2,4, \ldots$. The basic $\mathrm{ES}_{\mathrm{n}}$ quadrature sets are defined on the interval $(0,1)$ in the one-dimensional case and on the principal octant in the two-dimenstonal case. In the first instance, $\frac{1}{2 n}$ nodes $\mu_{m}$ are placed on the $\mu$-interval $(0,1), m=1,2, \ldots, M=$ $\frac{1}{2} \mathfrak{n}$, with weights $w_{m}$ which are sums of $\left(\frac{1}{2} n-m+1\right)$ point weights, and in the second instance $M(n)$ nodes $\Omega_{\mathrm{m}} \equiv\left(\mu_{\mathrm{m}}, n_{\mathrm{m}}, \xi_{\mathrm{m}}\right)$ on the principal octant (where the components of $\Omega$ are positive), $m=1,2, \ldots, M=$ $n(n+2) / 8$, with equal weights $w_{m}$,

$$
w_{m}=w=8 / n(n+2)
$$

Here $\Omega_{\mathrm{m}}$ may also be written $\left(\mu_{\ell, m}, n_{\ell, m}, \xi_{\ell, m}\right)$ in terms of a $\mu$-index $m, m=1,2, \ldots, \frac{1}{2 n}-\ell+1$, and 
a $\xi$ or level index $\ell, \ell=1,2, \ldots$, sn. The components are assumed to satisfy

$$
\mu_{\ell, \mathrm{m}}^{2}+n_{\ell, \mathrm{m}}^{2}+\xi_{\ell, \mathrm{m}}^{2}=1 .
$$

In the two-dimensional case, as indicated above, the octant is covered by a triangular pattern of equal areas $\frac{1}{2} \pi w_{\ell, m}=\frac{1}{2} \pi w, n(n+2) / 8$ in number, so that

$$
\Sigma_{\ell, \mathbb{m}} w_{\ell, \mathrm{m}}=1 \text {. }
$$

The one-dimensional weights $w_{m}$ moy be regarded as level weights, that is, sums of point weights by level

$$
w_{m}=\Sigma_{\ell} w_{\ell, \mathbb{R}}=\left(1_{2} \mathrm{n}-\mathrm{m}+1\right) w .
$$

Here $\frac{1}{2} \pi w_{m}$ correspond to areas of latitudinal bands on the octant. The weights given by Eq. (6.4) do not differ greatiy from the ordinary Gauss quadrature weights.

The quadrature nodes (or points) are also placed in a triangular fashion on the octant with one node per point weight and 1 in $-\ell+1$ nodes per level. They are placed in a symmetric pattern with respect to the three axes so as to form a closed set with respect to permutation of the components. Therefore, if $(\mu, n, \xi)$ is a node in the set so are $(\mu, \xi, \eta),(n, \xi, \mu),(n, \mu, \xi),(\xi, \mu, n)$, and $(\xi, \eta, \mu)$. Such groupings may consist of less than six points, three points if two of the components are equal, and ore point $(\sqrt{3} / 3, \sqrt{3} / 3, \sqrt{3}, 3)$ if a!l three are equal. Al together one can count $\left[\left(n^{2}+8 n+28\right) / 48\right\}$ groupings and $[n(n+2) / 24]$ independent components.

In $E Q_{n}$ quadrature, based on vertain assumptions discussed in Ref, 5, one is able to reduce the number of independent components to $[n(n+12) / 48]$. By correlating these with the same number of independent ever moments $\mathrm{M}_{k, j}, k+j \leq n$, une can set up a system of nonlinear equations for determining the In, set for any $n$.

because of the triangular symmetry, $\Omega_{\mathrm{m}}$ can be written $\left(\mu_{\ell, m}, \mu_{\ell, k}, \mu_{m, \ell}\right)$ where $k=i_{2 n}-\ell-m+2$. The permutation invariance combined with Eq. (6.2) implies that the $\mathrm{M}_{2,0}$ moment condition is satisfled.

$$
\mathrm{M}_{2,0}=\Sigma_{\ell, \mathrm{m}} \mathrm{w}_{\ell, \mathrm{m}} \mu_{\mathrm{n}, \mathrm{m}}{ }^{2}=1 / 3 .
$$

Extension of the basic quadrature from $(0,1)$ to $(-1,1)$ or from one to two, fuur, or eight octants, as a given problem may require, is defined in terms of symmetry about coordinate planes with the appropriate sign changes in direcition cosines. Because $M$ changes from $\frac{1}{2} n$ to $n$ or from $n(n+2) / 8$ to $n(n+2) / 4, n(n+2) / 2$, or $n(n+2)$, respectively, the weights are renormalized so that their sum remains at unity.

The one-dimensional $\mathrm{ES}_{\mathrm{n}}$ sets may be generated independently of the two-dimensional ones. This gives us simple means for describing the steps in the construction and examining some of the mure immediate consequences. In the first step the reights are selected, here

$$
w_{m}=\left(\frac{1}{2} n-m+1\right)[8 / n(n+2)] .
$$

In the second, certain $\mu$ coordinates are computed, the lower $\mu-i n t e r v a l$ boundaries $\mu_{m-\frac{1}{2}}$,

$$
\mu_{m-\frac{1}{2}}=1-\frac{1}{2}\left(\frac{1}{2} n-m+2\right) w_{m} \text {, }
$$

and the interval midpoint values $\bar{\mu}_{m}$,

$$
\vec{\mu}_{m}=\mu_{m-\frac{1}{2}}+1_{2} w_{m}=1-\frac{1}{2}\left(l_{2 n}-m+1\right) w_{m} .
$$

Here $\mu_{b_{2}}=0, \mu_{3 / 2}=w_{1}, \mu_{5 / 2}=w_{1}+w_{2}$, etc., and $\mu_{1}=\frac{1}{2} w_{1}, \mu_{2}=w_{1}+b_{2} w_{2}$, etc.

In the third step, a scaling process is applied to $\bar{\mu}_{\mathrm{m}}$. In method $I \mu_{\mathrm{m}}$ is written

$$
\mu_{\mathrm{m}}=\mathrm{R} \bar{\mu}_{\mathrm{m}}
$$

with $R$ determined so that

$$
\Sigma_{\mathrm{m}} \mathbf{w}_{\mathrm{m}} \mu_{\mathrm{m}}^{2}=1 / 3
$$

For transport work, a slightly different method (II) is considerably better. Here $\mu_{m}$ is written

$$
\mu_{m}=R \mu_{m-\frac{1}{2}}+\bar{\mu}_{m}
$$

with $R$ determined so that Eq. (6.10) is satisfied. This is the method selected here and the one examined below. Table VI-1 gives values of $R, R=R(n)$, for a sequence of $n$. For large $n, R$ appears to behave as $1 / \mathrm{n}^{2}$. The one-dimensional $\mathrm{ES}_{\mathrm{n}}$ sets, based 
TABLE VI-1

VALUES OF THE SCALE FACTOR $R$ VS $n$

\begin{tabular}{|c|c|}
\hline$\underline{\mathrm{n}}$ & $\mathbf{R}$ \\
\hline 4 & 0.0728757 \\
\hline 6 & 0.0287804 \\
\hline 8 & 0.0157065 \\
\hline 10 & 0.0099458 \\
\hline 12 & 0.0068776 \\
\hline 14 & 0.0050444 \\
\hline 16 & 0.0038600 \\
\hline 20 & 0.0024709 \\
\hline
\end{tabular}

on method $I I, n=2,4, \ldots, 16$, are included in Table VI-8.

In a first test of the quadrature, the linear extrapolation length $z_{0}$ is obtafned as a function of $n$. The accuracy of this quantity is importani to the accuracy of the results to a number of benchmark problems in transport theory. The exact value of $z_{0} 1 s$ known to be 0.71044609 . For finite $n, z_{0}$ may be obtained as the sum of the poles minus the sum of the zeroes of the following equation.

$$
\Sigma_{m} \frac{w_{m}}{1-\mu_{m}^{2} / \mu^{2}}-1=0 .
$$

The poles are clearly located at the quadrature nodes. There are $1 / 2 \mathrm{n}-1$ zeroes. For $\mathrm{n}=4$ one finds $\mu=\sqrt{3} \mu_{1} \mu_{2}$ to be the single zero, hence for $\mathrm{n}=4$

$$
z_{0}=\mu_{1}+\mu_{2}-\sqrt{3} \mu_{1} \mu_{2}
$$

Values of $z_{0}$ for several sequences of $n$ are given in Table VI-2. The errors in the $E S_{n}$-based values behave approximately as $-0.09 / \mathrm{n}^{2}$. Here $E S_{n}$ by scaling II represents a significant improvement over Gauss quadrature which, for $\mathrm{n}=4,8$, and 16 , gives $z_{0}=0.694025,0.706918$, and 0.709609 , respectively. $E S_{n}$ by scaling I gives similar values.

Incldentally, Gauss quadrature can be very closely simulated by the $\mathrm{ES}_{\mathrm{n}}$ technique with scaling I using in the first step either the exact Gauss weights or approximate values from $w_{m}=\mu_{m+\frac{1}{2}}-$ $\mu_{m-\frac{1}{2}}$ where

$$
\mu_{m-\frac{1}{2}}=\sin \frac{\pi}{2} \frac{2(m-1)}{n+\frac{1}{2}} / \sin \frac{\pi}{2} \frac{n}{n+\frac{1}{2}} .
$$

TABLE VI-2

THE EXTRAPOLATION LENGTH $z_{0}$ FOR $c=1$ AND ES $\mathbf{n}$ QUADRATURE

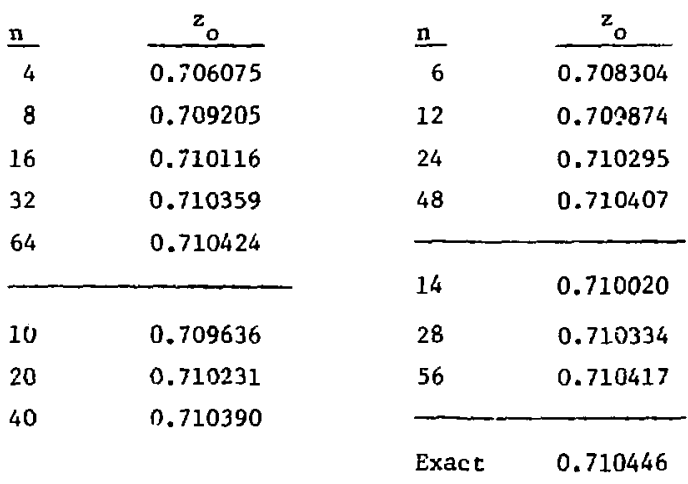

In a second test, the moments $M_{k}$ are computed from the $E S_{\mathfrak{n}}$ data and compared to the exact values, $M_{k}=1 /(k+1)$, for a sequence of $k$ for several values of $n$. For $k=0$ and 2 the $E S_{n}$ data give correct values by construction. Table VI-3 gives values of $M_{k}$ for $k=4,6,8,12$, and 16 , and $n=4,6,8,12$, 16,32 , and 64 . The errors for layge $n$ behave as $A_{k} / n^{2}$ where $A_{4} \approx 1.35, A_{6} \approx 1.94, A_{8} \approx 2.26$, and $A_{16}=2.80$. The coefficients $A_{k}$ appiar to be bounded with respect to k. In this test, Gauss quadrature is clearly superior giving exact values by definition for $M_{k}$ for even $k$ to $k^{*}=2 n-2$ inclusive. However, in $s_{n}$-type transport calculations, as far as has been observed, exactness for $k>2$ appears to have no clear advantages over $1 / n$ or faster convergence.

In a third test, values of the coefficients $2 \alpha_{m-\frac{1}{2}}$ of special importance in spherical geometry, are computed using

$$
2 \alpha_{m-\frac{1}{2}}=2 \Sigma_{\ell=\mathbb{m}}^{1 / m} w_{\ell} \mu_{\ell}
$$

and compared to the exact values from

$$
2 \alpha_{m-\frac{1}{2}}=1-\bar{\mu}_{m-\frac{1}{2}}{ }^{2} .
$$

As can be seen from the data in Table VI-4 and also confirmed analytically, there is rapid convergence of $2 \alpha_{\mathrm{m}-\frac{1}{2}}$ as obtained from Eq. (6.15) as $\mathrm{n}$ increases. 
TABLE VI-3

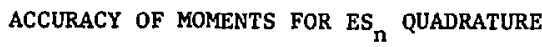

(zeroeth and second moments exact)

\begin{tabular}{|c|c|c|c|c|c|}
\hline Moment & 4 & 6 & 8 & 12 & 16 \\
\hline \multicolumn{6}{|l|}{$\mathbf{n}$} \\
\hline 4 & 0.209877 & 0.157750 & & & \\
\hline 6 & 0.204154 & 0.148843 & 0.117595 & & \\
\hline 8 & 0.202293 & 0.146140 & 0.114841 & 0.080968 & \\
\hline 12 & 0.200998 & 0.144286 & 0.112766 & 0.078794 & 0.060774 \\
\hline 16 & 0.200554 & 0.143651 & 0.112035 & 0.077979 & 0.059940 \\
\hline 32 & 0.200135 & 0.143051 & 0.111337 & 0.077184 & 0.059102 \\
\hline 64 & 0.200033 & 0.142905 & 0.111167 & 0.076987 & 0.058892 \\
\hline Exact & 0.200000 & 0.142857 & 0.111111 & 0.076923 & 0.058824 \\
\hline
\end{tabular}

TABLE VI-4

VALUES OF $2 \alpha_{\mathrm{m}-\frac{1}{2}}$ FOR SELECTED $\mathrm{n}$

$\begin{array}{lll}\underline{\mathrm{n}=4} & \frac{\text { Approx }}{\alpha_{\frac{1}{2}}} & \frac{\text { Exact }}{1.0324} \\ \alpha_{3 / 2} & 0.5879 & 1.0000 \\ \underline{\mathrm{n}=8} & & 0.5556 \\ \alpha_{\frac{1}{2}} & 1.0110 & 1.0000 \\ \alpha_{3 / 2} & 0.8510 & 0.8400 \\ \alpha_{5 / 2} & 0.5172 & 0.5100 \\ \alpha_{7 / 2} & 0.1928 & 0.1900\end{array}$

Approx

Exact

1.0033

1.0000

$\alpha_{3 / 2}$

0.9539

0.9506

$\alpha_{5 / 2}$

0.8293

0.8264

$\alpha_{7 / 2}$

0.6621

0.6597

$\alpha_{9 / 2}$

0.4802

0.4784

$\alpha_{11 / 2}$

0.3067

0.3056

$\alpha_{13 / 2}$

0.1603

0.1597

$\alpha_{15 / 2}$

0.0550

0.0548

The two-dimensional ES $\mathrm{n}$ quadrature sets are also obtained by a three-step method as follows.

1. The principal octant is divided into $n(n+2) / 8$ mesh cells of equal area located in a triangular and symmetric manner as discussed earlier. The associated mesh points, also invariant with respect to nermutation of components, can be determined from certain trigonometric equations. The mesh division is illustrated in Fig. 6.1 for $\mathrm{n}=16$. Note the successive $[(n+2) / 6]$ annular regions containing $3\left(\frac{1}{2} n-1\right), 3\left(\frac{1}{2} n-4\right), \ldots$, equal areas.

2. The midpoint conrdinates $\bar{\mu}_{m}, \bar{\eta}_{m}$, and $\bar{\xi}_{m}$ are computed for each mesh cell. These coordinates, which may also be written $\bar{\mu}_{\ell, m}, \bar{\mu}_{\ell, k}$, ard $\bar{\mu}_{m, \ell}$ $\left(k=\frac{1}{2} n-\ell-m+2\right)$, can also be found from analytica1 formulas.

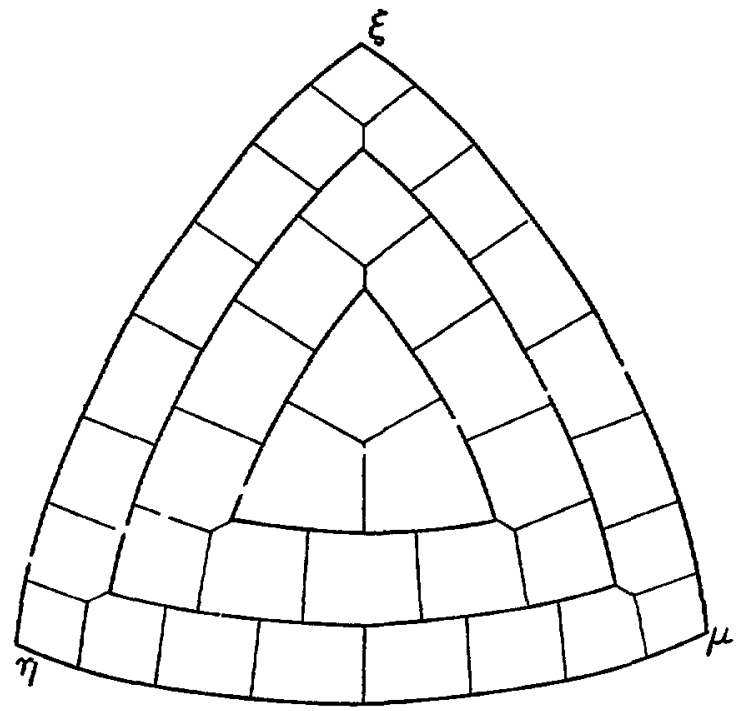

Fig. 6.1. Mesh division for $\mathrm{n}=16$. 
3. The midpoint coordinatiss are scaled using

$$
\mu_{\ell, m}=\mathbf{R} \mu_{m-\frac{1}{2}}+\vec{\mu}_{\ell, \pi}
$$

with $\mu_{m-1 / 2}$ from $\mathrm{Eq} .(6.7)$ and $R$ determined for each grouping of points so that

$$
\begin{aligned}
& \left(R \mu_{m-\frac{1}{2}}+\bar{\mu}_{\ell, \mathbb{R}}\right)^{2}+\left(R \mu_{k-1 / 2}+\bar{\mu}_{\ell, k}\right)^{2} \\
& +\left(\mathrm{R} \mu_{\ell-l_{2}}+\bar{\mu}_{\mathrm{m}, \ell}\right)^{2}=1 \text {. }
\end{aligned}
$$

The formulas and procedure in $\mathrm{ES}_{\mathrm{n}}$ quadrature will be discussed in more detall in a future report.

As a first test of two-dimensional quadrature, a selection of moments $M_{k, o}$ is calculated and presented in Table VI-5 to be compared to data in Table VI-3. As shown, the agreement with exact values is better in the two-dimensional case.

In a second test, comparison of three variations of $E S_{n}$ with $E Q_{n}$ are made. Table VI-6 gives $\mu_{\ell, m}$ for $n=4,8$, and 16 for a previous version of $E S_{n}(A)$ and the present version (B) with scaling I as well as for (B) with scallng II. In all cases the agreement is very close which may be taken as a confirmation of the reasonableness of all these quadratures, $\mathrm{EQ}_{\mathrm{n}}$ included.

Method $\mathrm{A}$ is based on a simple half-symmetric division of the octant, splitting bands defined by $\left(\xi_{\lambda-\frac{1}{2}}, \xi_{\ell+\frac{1}{2}}\right), \ell=1,2, \ldots, \frac{1}{2} n$, into $\frac{1}{2} n-l+1$ equal sectors for a total of $n(n+2) / 8$ sectors (mesh cells) where the $\xi^{\prime}$ 's are taken from Eq. (6.7). After computing the midpoint coordinates $\vec{\mu}_{\ell, m}$,

\begin{tabular}{|c|c|c|c|c|c|c|}
\hline & & & & & & \\
\hline & $\underline{\ell}$ & 틀 & $\frac{E Q_{n}}{(L A-473)}$ & $\begin{array}{l}\text { Hethad } A \\
\text { Sealing I }\end{array}$ & $\begin{array}{l}\text { Kethod B } \\
\text { 5coling I }\end{array}$ & $\begin{array}{l}\text { Te:hod } \\
\text { Scaling II }\end{array}$ \\
\hline$n=4$ & $I$ & 1 & 0.3500212 & 0.3505622 & 0.1696826 & 0.3344052 \\
\hline & 1 & 2 & 0.8688903 & 0.8684540 & 0.8692629 & 0.8811052 \\
\hline$\underline{n-8}$ & 4 & 1 & 0.1971380 & 0.1944296 & 0.1957928 & 0.1932356 \\
\hline & 3 & 1 & 0.2133982 & 0.2137991 & 0.2203029 & 0.7075330 \\
\hline & 3 & 2 & 0.5512958 & 0.5473098 & 0.5503087 & 0.5497634 \\
\hline & $=$ & $\because$ & nestient & n xaracas & ก ธร7งรก) & n \\
\hline & 2 & 3 & 0.8065570 & 0.8091612 & 0.8080430 & 0.8091294 \\
\hline & 1 & 4 & 0.9603506 & 0.9614543 & 0.9609008 & 0.9619356 \\
\hline$n=16$ & B & 1 & 0.1050159 & 0.1029311 & 0.1040726 & 0.1036946 \\
\hline & 7 & 1 & 0,1152880 & 0.1130805 & 0.1137610 & 0.1133221 \\
\hline & 6 & 1 & 0.1152880 & 0.1260605 & 0.1145198 & 0.1140958 \\
\hline & 5 & 1 & 0.1152880 & 0.1172276 & 0.1145198 & 0.1160958 \\
\hline & 7 & 2 & 0.3016701 & 0.3009446 & 0.3005210 & 0.3002697 \\
\hline & 6 & 2 & 0.3284315 & 0.32385564 & 0.3266520 & 0.3263778 \\
\hline & 5 & 2 & 0.3332906 & 0.3329597 & 0.3315867 & 0.3313218 \\
\hline & 4 & 2 & 0.3332906 & 0.3355648 & 0.3325955 & 0.3323335 \\
\hline & 6 & 3 & 0.4763525 & 0.4774090 & 0.4750315 & 0.4749405 \\
\hline & 3 & 3 & $0.51073: 4$ & 0.5085062 & 0.5121306 & 0.5120317 \\
\hline & 4 & 3 & 0.5315431 & 0.5210090 & 0.5205894 & 0.5205236 \\
\hline & 5 & 4 & 0.6327389 & $0.632997 \mathrm{u}$ & 0.6321597 & 0.6 .721909 \\
\hline & 4 & 4 & 0,6666774 & 0.6661117 & 0.666 A509 & 0.6669162 \\
\hline & 3 & 4 & 0.6752631 & 0.6760911 & 0.6767373 & 0.6768386 \\
\hline & 4 & 3 & 9.7657351 & 0.7652271 & 0.7553038 & 0.7663650 \\
\hline & 3 & $s$ & 0.7925089 & 0.7940776 & 0.7923216 & 0.1924957 \\
\hline & 3 & b & 0.8727534 & 0.8709825 & 0.8726851 & $0.8 \div 25902$ \\
\hline & 2 & 6 & 0.8835877 & $0.8 B 89657$ & $0.8 B f 9030$ & 0.8871049 \\
\hline & 2 & 7 & 0.9464163 & 0.9469335 & 0.9469665 & 0.9470501 \\
\hline & 1 & 8 & 0.9889102 & 0.9893381 & 0.9891096 & 0.9591890 \\
\hline
\end{tabular}

TABLE VI-6 VALUES OF $\mu_{\ell, m}$ FOR VARIOUS METHODS

$\bar{n}_{\ell, m}, \bar{\xi}_{\ell, \mathrm{m}}$ for each cell, the following symmetrizing operation is performed before scaling

$$
\frac{1}{3}\left(\bar{\mu}_{\ell, m}+\bar{\mu}_{k, m}+\bar{\xi}_{m}\right)+\bar{\mu}_{\ell, m}
$$

As a third test, the values of $n_{\ell, \frac{2}{2}}$ are determined, of special significance in cylindricai geumetry, where

TABLE VI-5

ACCURACY OF MOMENTS $M_{k, 0}$ for ES ${ }_{n}$ TWO-DIMENSIONAI CASE

$\begin{array}{cccccc}\frac{\mathrm{k}}{\mathrm{n}} & \underline{4} & \underline{6} & \underline{8} & \underline{12} & \\ 4 & 0.209241 & 0.156904 & 0.115398 & & \\ 6 & 0.203230 & 0.147185 & 0.112958 & 0.078808 & \\ 8 & 0.201376 & 0.144602 & 0.111954 & 0.077764 & 0.059632 \\ 12 & 0.200632 & 0.143655 & 0.111552 & 0.077390 & 0.059295 \\ 16 & 0.200325 & 0.143266 & 0.111217 & 0.077038 & 0.058943 \\ 32 & 0.200077 & 0.142954 & 0.111137 & 0.076952 & 0.058854 \\ 64 & 0.200019 & 0.142881 & 0.111111 & 0.076923 & 0.058824\end{array}$




$$
\eta_{\ell, \frac{1}{2}}=\left(\pi / 2 w_{\ell}\right) \alpha_{\ell, \frac{1}{2}}
$$

given exactly by

$$
n_{l, \underline{1}}=\sqrt{1-\xi_{\ell}^{2}}=\sqrt{1-\mu_{l}^{2}}
$$

with $\mu_{\ell}^{2}$ obtained from

$$
\mu_{\mathrm{m}}^{2}=\left(\Sigma_{\ell} w_{\ell, \mathrm{m}} \nu_{\ell, \mathrm{m}}^{2}\right) / w_{\mathrm{m}} .
$$

and given approximately by

$$
n_{\ell, \ell}=\left(\pi w / 2 w_{\ell}\right) \Sigma_{m} \mu_{\ell, m}
$$

The differences between approximate and exact values for $\eta_{\ell, \ell}$ are given in Table vI-7 for $n=4,6$, $\ldots, 16$, and $\hat{\chi}=1,2, \ldots, 1,2 n$. Also, in the first column, the average absolute error is given, a weighted average with $w_{\ell}$ as weights. Similar tables have been constructed for $\eta_{\ell, 3 / 2}, \eta_{6,5 / 2}$, etc., giving simflar results.

Table VI-7 is only mildly reassuring in regard to convergence with $n$. However, this is not as much a test of the quadrature as a cest of the numertcal procedure for handling direction-to-direction transfer by streaming in cylindrical geometry. The procedure is built on the assumption of withinlevel transfer only, the basis for Eq. (6.23). Further tests are indicated here.

Table VI-8 gives one-dimensional and two-dimenslonal Es $n$ quadrature sets for $n=4,6, \ldots, 16$. In the one-dimensional case $u_{m}$ and two sets of nodes $\mu_{m}$ are given, the first based on Eq. (6.11) and the second on Eq. $(6.22)$.

\section{RFFERENCES}

1. Bengt G. Carlson, "Modifled Difference Schemes for the Transport Equation Solution," Los Alamas Scientif ic Laburatory report, LA-5760 (1974).

2. 1. P. Grant, "Superical Anelysis of Discrete Ordinates Methods," J. Comput. Phys. 2, No. 4 (1968).

3. Wir. H. Reed and K. D. Lachrop, "Truncation Error Analysig of Finice Difference Appro" inatjons to the Transport Equation," Nucl. Sci. Eng. 41. No. 2, 237 (1970).

4. B. C. Carlson, "Transport Theory: Discrete ordinates Quadrature Over the Unit Sphere," Los Alamos Sclentific Laboratory report. IA4554 (1970).

5. B. C. Farlson, "Tables of Symmetric Equal Weight Quadrature $E \mathrm{Q}_{\mathrm{n}}$ Over the Unit Sphere," Los Alamos Scientific Laboratory report. LA-

\begin{tabular}{|c|c|c|c|c|c|c|c|c|c|}
\hline$\underline{n}$ & $\begin{array}{l}\text { Average } \\
\text { Erroz }\end{array}$ & $2=1$ & $2=2$ & $2=3$ & $2=4$ & $2=5$ & $l=6$ & $2=7$ & $8=8$ \\
\hline 4 & 0.0256 & 0.0122 & 0.0524 & & & & & & \\
\hline 6 & 0.0143 & 0.0089 & 0.0104 & 0.0384 & & & & & \\
\hline$B$ & 0.0091 & 0.0076 & 0.0055 & 0.0071 & 0.0303 & & & & \\
\hline 10 & 0.0062 & 0.0066 & 0.0045 & 0.0021 & 0.0057 & 0.0249 & & & \\
\hline 12 & 0.0046 & 0.0059 & 0.0048 & 0.0009 & 0.0006 & 0.0049 & 0.0212 & & \\
\hline 14 & 0.0038 & 0.0054 & 0.0053 & 0.0011 & -0.0012 & -0.0001 & 0.0043 & 0.0184 & \\
\hline 16 & 0.0036 & 0.0049 & 0.0057 & 0.0016 & -0.0013 & -0.0021 & -0.0003 & 0.0039 & 0.00162 \\
\hline
\end{tabular}
4734 (1971).

TABLE VI-7

ERROR IN $n_{\ell, \frac{1}{2}}$ FOR ES $n$ AND SELECTED $n$ 
QUADRATURF. SFTS

\begin{tabular}{|c|c|c|c|c|c|}
\hline $\begin{array}{l}\text { 田 } \\
n \times 4\end{array}$ & $\underline{1}$ & $\underline{2}$ & 3 & 4 & $\underline{5}$ \\
\hline$\omega_{n t}$ & 0.6666667 & 0.3333333 & & & \\
\hline i & 0.3344052 & 0.8819171 & & & \\
\hline " & 0.3333333 & 0.8819171 & & & \\
\hline $2, m$ & 0.3344052 & & & & \\
\hline$n 1, n$ & 0.3344052 & 0.8811052 & & & \\
\hline \multicolumn{6}{|l|}{$\underline{n-6}$} \\
\hline$w_{m}$ & 0.5000000 & 0.2 .333333 & 0.1666667 & & \\
\hline$m$ & 0.2500000 & 0.6810569 & 0.9406503 & & \\
\hline 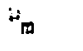 & 0.2508939 & 0.6825427 & 0.9377780 & & \\
\hline $3, \mathrm{~m}$ & 0.2455325 & & & & \\
\hline$H_{2, \pi}$ & 0.2612869 & 0.6825427 & & & \\
\hline H, D & 0.2455325 & 0.6825427 & 0.9377780 & & \\
\hline \multicolumn{6}{|l|}{$n=8$} \\
\hline$w_{m}$ & 0.4000000 & 0.3000000 & 0.2000000 & 0.1000000 & \\
\hline in & 0.2000000 & 0.5562826 & 0.8109946 & 0.9641359 & \\
\hline$i n$ & 0.2005118 & 0.5591103 & 0.8091294 & 0.9619356 & \\
\hline$H_{4, m}$ & 0.1932356 & & & & \\
\hline $\mathrm{H}_{3}, \mathrm{~m}$ & 0.2075330 & 0.5497634 & & & \\
\hline$H_{2, m}$ & 0.2075330 & 0.5773503 & 0.8091294 & & \\
\hline$\mu_{1, m}$ & 0.1932356 & 0.5497634 & 0.8091294 & 0.9619356 & \\
\hline \multicolumn{6}{|l|}{$n=10$} \\
\hline$u_{m}$ & 0.3333333 & 0.2666667 & 0.2000000 & 0.1333333 & 0.0666667 \\
\hline$u_{m}$ & 0.1666667 & 0.4699819 & 0.7059675 & 0.8746233 & 0.9759494 \\
\hline$\mu_{m}$ & 0.1671001 & 0.4717345 & 0.7066294 & 0.8728505 & 0.9743667 \\
\hline$H_{5, m}$ & 0.1590747 & & & & \\
\hline$H_{4, \pi}$ & 0.1717616 & 0.4567603 & & & \\
\hline$\mu_{3, m}$ & 0.1732011 & 0.4862478 & 0.6964199 & & \\
\hline$\mu_{2, m}$ & 0.1717616 & 0.4862478 & 0.7260345 & 0.8728505 & \\
\hline$\mu_{1, m}$ & 0.1590747 & 0.4567603 & 0.6964199 & 0.8728505 & 0.9743667 \\
\hline
\end{tabular}


TABLE VI-8 (cont)

\begin{tabular}{|c|c|c|c|c|c|c|c|c|}
\hline $\begin{array}{l}m \\
\underline{n}=12 \\
\end{array}$ & 1 & $\underline{2}$ & $\underline{3}$ & 4 & $\underline{\mathbf{5}}$ & $\underline{6}$ & $\underline{7}$ & $\underline{8}$ \\
\hline$w_{m}$ & 0.2857143 & 0.2380952 & 0.1904762 & 0.1428571 & 0.0952381 & 0.0476190 & & \\
\hline$\mu_{m}$ & 0.1428571 & 0.4067269 & 0.6226502 & 0.7906269 & 0.9106570 & 0.9827406 & & \\
\hline$H_{m}$ & 0.1432328 & 0.4082299 & 0.6231948 & 0.7902040 & 0.9092277 & 0.9815823 & & \\
\hline${ }^{L} 6, m$ & 0.1350855 & & & & & & & \\
\hline$: 5, m$ & 0.1465973 & 0.3896334 & & & & & & \\
\hline$H 4, m$ & 0.1476756 & 0.4185322 & 0.6053009 & & & & & \\
\hline$H_{3, m}$ & 0.1476756 & 0.4234281 & 0.6405890 & 0.7821782 & & & & \\
\hline $\mathrm{H}_{2}$, 四 & $0.1 \AA 65973$ & 0.4205322 & 0.6405890 & 0.8060158 & 0.9092277 & & & \\
\hline$H_{1, m}$ & 0.1350855 & 0.3896334 & 0.6053069 & 0.7821782 & 0.9092277 & 0.9815823 & & \\
\hline \multicolumn{9}{|l|}{$n=14$} \\
\hline$w_{m}$ & 0.2500000 & 0.2142857 & 0.1785714 & 0.1428571 & 0.1071429 & 0.0714286 & 0.0357143 & \\
\hline$t_{m}$ & 0.1250000 & 0.3584040 & 0.5559135 & 0.7175286 & 0.8432492 & 0.9330754 & 0.9870071 & \\
\hline$\omega$ & 0.1253259 & 0.3596677 & 0.5573845 & 0.7167122 & 0.8425374 & 0.9319493 & 0.9861350 & \\
\hline$\omega_{7, m}$ & 0.1173407 & & & & & & & \\
\hline $6, \mathrm{mn}$ & 0.1278521 & 0.3392997 & & & & & & \\
\hline$\mu_{5, \mathrm{~m}}$ & 0.1287323 & 0.3669064 & 0.5329202 & & & & & \\
\hline$H_{4, \pi}$ & 0.1287323 & 0.3719382 & 0.5709843 & 0.7012232 & & & & \\
\hline$H_{3}, m$ & 0.1287323 & 0.3719382 & 0.5773503 & 0.7318735 & 0.8363158 & & & \\
\hline$\mu_{2, m}$ & 0.1278521 & 0.3669064 & 0.5709843 & 0.7318735 & 0.8548447 & 0.9319493 & & \\
\hline$\mu_{t, m}$ & 0.1173407 & 0.3392997 & 0.5329202 & 0.7012232 & 0.8363158 & 0.9319493 & 0.9861350 & \\
\hline \multicolumn{9}{|l|}{$\underline{n}=16$} \\
\hline$w_{m}$ & 0.2222222 & 0.1944444 & 0.1666667 & 0.1388889 & 0.1111111 & 0.0833333 & 0.0555556 & 0.0277778 \\
\hline$u_{m}$ & 0.1111111 & 0.3203022 & 0.5016083 & 0.6550294 & 0.7805655 & 0.8732166 & 0.9479828 & 0.9898639 \\
\hline$\mu_{n}$ & 0.1113942 & 0.3214668 & 0.5028882 & 0.6552855 & 0.7795403 & 0.8774551 & 0.9470901 & 0.8981890 \\
\hline$\mu_{8, m}$ & 0.1036946 & & & & & & & \\
\hline$\mu_{7, \mathrm{~m}}$ & 0.1133421 & 0.3002897 & & & & & & \\
\hline$\mu_{6, m}$ & 0.1140958 & 0.3263778 & 0.4749405 & & & & & \\
\hline$\mu_{5, m}$ & 0.1140958 & 0.3313218 & 0.5120317 & 0.6321909 & & & & \\
\hline$\nu_{4, m}$ & 0.1140958 & 0.3323335 & 0.5205236 & 0.6669162 & 0.7663660 & & & \\
\hline$\mu_{3, m}$ & 0.1140958 & 0.3313218 & 0.5205236 & 0.6768386 & 0.7924957 & 0.8725902 & & \\
\hline$\mu_{2, \mathrm{~m}}$ & 0.1133421 & 0.3263778 & 0.5120317 & 0.6669162 & 0.7924957 & 0.8871049 & 0.9470901 & \\
\hline$\mu_{1, m}$ & 0.1036946 & 0.3002897 & 0.4749405 & 0.6321909 & 0.7663660 & 0.8725902 & 0.9470901 & 0.9891890 \\
\hline
\end{tabular}

